ARTICLE

Received 19 Feb 2014 | Accepted 2 Oct 2014 | Published 3 Dec 2014

\title{
Pressure-temperature evolution of primordial solar system solids during impact-induced compaction
}

\author{
P.A. Bland', G.S. Collins², T.M. Davison², N.M. Abreu ${ }^{3}$, F.J. Ciesla4 ${ }^{4}$ A.R. Muxworthy ${ }^{2}$ \& J. Moore ${ }^{2}$
}

Prior to becoming chondritic meteorites, primordial solids were a poorly consolidated mix of $\mathrm{mm}$-scale igneous inclusions (chondrules) and high-porosity sub- $\mu \mathrm{m}$ dust (matrix). We used high-resolution numerical simulations to track the effect of impact-induced compaction on these materials. Here we show that impact velocities as low as $1.5 \mathrm{~km} \mathrm{~s}^{-1}$ were capable of heating the matrix to $>1,000 \mathrm{~K}$, with pressure-temperature varying by $>10 \mathrm{GPa}$ and $>1,000 \mathrm{~K}$ over $\sim 100 \mu \mathrm{m}$. Chondrules were unaffected, acting as heat-sinks: matrix temperature excursions were brief. As impact-induced compaction was a primary and ubiquitous process, our new understanding of its effects requires that key aspects of the chondrite record be re-evaluated: palaeomagnetism, petrography and variability in shock level across meteorite groups. Our data suggest a lithification mechanism for meteorites, and provide a 'speed limit' constraint on major compressive impacts that is inconsistent with recent models of solar system orbital architecture that require an early, rapid phase of main-belt collisional evolution.

\footnotetext{
${ }^{1}$ Department of Applied Geology, Curtin University, GPO Box U1987, Perth, Western Australia 6845, Australia. ${ }^{2}$ Impacts \& Astromaterials Research Centre (IARC), Department of Earth Science \& Engineering, Imperial College London, South Kensington Campus, London SW7 2AZ, UK. ${ }^{3}$ Earth Science Program, Pennsylvania State University-Du Bois Campus, Du Bois, Pennsylvania 15801, USA. ${ }^{4}$ Department of Geophysical Science, University of Chicago, 5734 South Ellis Avenue, Chicago, Illinois 60430, USA. Correspondence and requests for materials should be addressed to P.A.B. (email: p.a.bland@curtin.edu.au).
} 
C hondritic asteroids are among the most primitive objects in the solar system, containing a unique record of solar system formation. Three processes dominated their evolution as geological bodies - thermal metamorphism, aqueous alteration and impacts. Meteorite classification schemes are based on the degree to which a given sample was exposed to these processes. The impact record in chondrites has been studied in detail ${ }^{1}$. Shock metamorphism is not considered a dominant factor in the evolution of the most primitive meteorites, the carbonaceous chondrites (CC), as $85 \%$ are ranked S1 ('unshocked'; <4-5 GPa) or S2 ('very weakly shocked'; $5-10 \mathrm{GPa})^{1,2}$. Associated low-intensity collisions are thought to leave a chondrite essentially unscathed, with no effects from local pressure-temperature (PT) excursions, and minimal post-shock thermal metamorphism ${ }^{1}$. Primitive (that is, unequilibrated) ordinary chondrites rank at higher shock levels: $50 \%$ are S3 ('weakly shocked'; $10-20 \mathrm{GPa})^{1}$. Shock level is calibrated against shock recovery experiments on non-porous or lowporosity crystals and rocks ${ }^{1}$, but porous objects respond very differently to impact than non-porous objects ${ }^{3,4}$. When shocking a porous material, extra $P \mathrm{~d} V$ work is expended to crush out the pore space. After release from the shocked state, that extra work (termed the 'waste heat') heats the material, thus porous material will reach higher temperatures than a non-porous material in a similar impact. Although they are now lithified (often low porosity) rocks, chondrite precursors were highly porous $^{5-8}$.

In addition, chondrites are bimodal materials: zeroporosity $\mathrm{mm}$-sized spherical chondrules set in an aggregate composed of sub- $\mu \mathrm{m}$ monomers. But the level of impact processing in chondrites is determined by shock metamorphic textures in large $(>50-100 \mu \mathrm{m})$ grains (for example, chondrule olivines), accessible via optical microsocpy ${ }^{1,2}$. The assumption has been that if chondrules are unshocked, so is matrix. The possibility that the shock record might be different in matrix has not been considered, and with notable exceptions ${ }^{9-11}$, the extent of shock metamorphism in matrix has not been explored.

Alongside petrographic observations and experimental impact studies, computational modelling has been utilized extensively to understand impact shock, generally applied to non-porous targets, but most recently in porous planetesimals ${ }^{11}$. In these models, porosity is parameterized, and derived PT estimates are 'bulk' values, averaged over large (asteroidal) scales, making it difficult to translate model predictions to meteorite observations. No numerical studies have attempted to resolve shock in the bimodal materials that were chondritic precursors. This is unfortunate given that they were arguably the starting point for all inner solar system objects.

An obstacle to understanding impact effects in porous meteorites has been the absence of direct information on the nature of the porosity prior to the shock event ${ }^{3}$. But a methodology that quantitatively relates rock fabric intensity to net compression ${ }^{7}$ has overcome this obstacle, allowing us to reconstruct a pre-compaction porosity for matrix and for the parent body as a whole. As outlined in that work ${ }^{7}$, it is possible to reconstruct a pre-compaction matrix porosity by deriving the ratio of final (that is, post-compaction) lengths to initial (precompaction) lengths of notional lines perpendicular to the matrix uniaxial fabric. This ratio $(Z)$ is a quantitative measure of the degree of compaction. Given the current observed (final) abundance of matrix $\left(A_{\mathrm{mf}}\right.$ ), and current (final) bulk porosity $\left(\phi_{\text {bf }}\right)$, we can estimate current (final) matrix porosity $\left(\phi_{\mathrm{mf}}\right)$ (assuming chondrule porosity $\sim 0 \%$ ). Given $Z$ and $\phi_{\mathrm{mf}}$, the initial (pre-compaction) matrix porosity $\left(\phi_{\mathrm{mi}}\right)$ is given by $\phi_{\mathrm{mf}} Z+(1-Z)$. It follows that initial bulk porosity $\left(\phi_{\mathrm{bi}}\right)$ is given by

$$
\phi_{\mathrm{bi}}=\frac{A_{\mathrm{mf}} \phi_{\mathrm{mi}}}{\left[A_{\mathrm{mf}} \phi_{\mathrm{mi}}+\left(1-\phi_{\mathrm{bf}}\right) Z\right]}
$$

and initial matrix abundance $\left(A_{\mathrm{mi}}\right)$ by $\phi_{\mathrm{bi}} / \phi_{\mathrm{mi}}$.

Thus far the early evolution of the material properties of solar system solids has been explored largely via experiments with analogue materials ${ }^{5}$. Most recently, impact compaction experiments compared final bulk porosities with chondrite values ${ }^{8}$. The saturation limit of the porosity varies for different materials: $0.68-0.42$ at a pressure of $1 \mathrm{MPa}^{8}$. The upper bound appears to be a closer approximation to $\phi_{\mathrm{mi}}$ for chondrite parent bodies. In OCs, $\phi_{\text {bf }}$ is typically $\sim 5 \%$ (ref. 12), but there are outliers with values up to $21 \%$ (ref. 13). For the most porous OCs it has been shown that this is primordial (not fracture) porosity ${ }^{13}$. Similarly, for anhydrous CCs there is a large range: $\phi_{\mathrm{bf}}=7-28 \%$ for CO chondrites, and $4-28 \%$ for $\mathrm{CV}$ chondrites ${ }^{14}$. Using the relation outlined above, assuming (conservatively), no compaction $\left(Z=1\right.$, and $\left.\phi_{\mathrm{mf}}=\phi_{\mathrm{mi}}\right)$, it is apparent that current matrix porosity $\left(\phi_{\mathrm{mf}}\right)$ in the most porous OCs must be $60-70 \% ; 60 \%$ in the most porous CVs and $66-70 \%$ in the most porous COs. In the CV chondrite Allende, where a detailed rock fabric analysis was performed ${ }^{7,15}$, and consistent matrix fabrics observed across the sample, degree of compaction consistent with $Z=\sim 0.5$ was defined $^{7}$ (that is, compaction had roughly halved the volume of the matrix aggregate). A $\phi_{\mathrm{mi}}$ value of $70-80 \%$ was derived ${ }^{5}$. The data from compaction experiments ${ }^{8}$, fabric analysis ${ }^{7}$ and an overview of literature porosity, appear consistent with $\phi_{\mathrm{mi}}=\sim 70 \%$ across most chondrite groups.

If chondrite precursors were initially highly porous, how were they compacted to the (generally) low-porosity rocks that we see today? Recent studies ${ }^{8}$ favour impacts as a compaction mechanism, and several other observations support that conclusion. Lithostatic pressure (gravitational compression) was trivial $(\sim 1 \mathrm{MPa}$ at the centre of a $100-\mathrm{km}$ radius asteroid, equivalent to a depth of $10 \mathrm{~m}$ on Earth ${ }^{16}$ ), indicating that it was not a significant factor in chondrite compaction. In addition, gravitational compaction would generate parent bodies with meteorites from the interior being more compacted (and showing more thermal metamorphism), than meteorites sourced from nearer the surface of the object. This is not observed. There is a lack of positive correlation between compaction and thermal metamorphism in $\mathrm{OCs}^{12}, \mathrm{COs}^{14}$ or $\mathrm{CVs}^{14}$. In the $\mathrm{CVs}$ there is a negative correlation: unmetamorphosed $\mathrm{CV}_{\mathrm{r}} \mathrm{s}$ are highly compacted (3.6\% porosity), while more metamorphosed $\mathrm{CV}_{\mathrm{o}} \mathrm{s}$ are not (averaging 19.7\%) ${ }^{14}$. Taken together, these observations are consistent with impact-induced compaction of initial highporosity material occurring on large (parent body?) scales.

Here we use numerical modelling to study the effects of impact-induced compaction on primordial chondritic materials. Our goal was to achieve sufficient resolution in simulations to allow a comparison with meteorite petrography observed in a thin section $(100 \mu \mathrm{m})$. Although low velocity impacts are expected to produce minor effects in $\mathrm{CCs}^{1,2}$, resolving at this finer 'mesoscale', we find that this is not the case. Even at $1.5 \mathrm{~km} \mathrm{~s}^{-1}$, porous matrix can experience peak $T>1,000 \mathrm{~K}$ as it compacts. PT varies by $>10 \mathrm{GPa}$ and $>1,000 \mathrm{~K}$ over $\sim 100 \mu \mathrm{m}$. Chondrules act as heat-sinks, so temperature excursions are brief. These results have implications for key aspects of the chondrite record, and provide a 'speed limit' constraint on the initial collisional evolution of planetesimals.

\section{Results}

Mesoscale simulations. We have developed a two-dimensional (2D) 'mesoscale' numerical approach that allows quantification of 
Table 1 | Results of mesoscale iSALE simulations of planar shock propagation through a chondrule/matrix mixture.

CC-like simulations (70\% initial matrix)

OC-like simulations (33\% initial matrix)

Impact

velocity
$\left(\mathrm{km} \mathrm{s}^{-1}\right)$

Initial matrix

fraction $\left(A_{\mathrm{mi}}\right)$

Initial matrix

porosity $\left(\varphi_{\mathrm{mi}}\right)$

Initial bulk

porosity $\left(\varphi_{\mathrm{bi}}\right)$

Bulk shock

pressure ${ }^{\star}$

Bulk shock

pressure $1 \sigma$

$(\mathrm{GPa})$

Bulk $T$ (final $)^{\dagger}$

Final bulk

porosity $\left(\varphi_{\text {bf }}\right)$

Final matrix

porosity $\left(\varphi_{\mathrm{mf}}\right)$

Final matrix

fraction $\left(A_{\mathrm{mf}}\right)$

Matrix

$T$ (final ${ }^{\ddagger}$ )

mean (K)

$T$ (final ${ }^{\ddagger}$ )

$1 \sigma(\mathrm{K})$

Matrix

$P\left(\right.$ peak $\left.^{\S}\right)$

mean (GPa)

Matrix

$P\left(\right.$ peak $\left.^{\S}\right)$

$1 \sigma(\mathrm{GPa})$

Matrix

$T$ (peak)

mean (K)

Matrix $T$

(peak) $1 \sigma(\mathrm{K})$

Chondrule

$T$ (final)

mean $(K)$

Chondrule $T$

(final) $1 \sigma(\mathrm{K})$

Chondrule $P$

(peak) mean

(GPa)

Chondrule

$P$ (peak)

$1 \sigma$ (GPa)

Chondrule

$T$ (peak)

mean (K)

Chondrule $T$

(peak) $1 \sigma(\mathrm{K})$

Matrix melt

fraction"

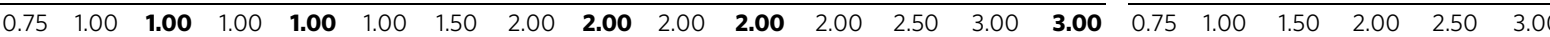

$0.70 \quad 0.70 \quad 0.70$

$0.70 \quad 0.60 \quad 0.60$

$0.490 .42 \quad 0.41$

$\begin{array}{lll}0 & 0.70 & \mathbf{0 . 7 0}\end{array}$

$\begin{array}{lll}0.70 & 0.70 & 0.70\end{array}$

$\begin{array}{lll}\mathbf{0 . 7 0} & 0.70 & \mathbf{0}\end{array}$

$\begin{array}{lll}0.70 & 0.70 & 0.70\end{array}$

$70 \quad 0.70$

$0.33 \quad 0.33$

0.330 .33

$33 \quad 0.33$

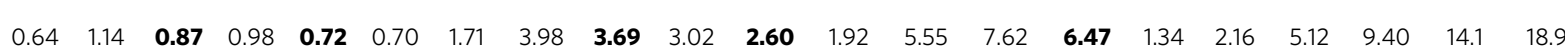

$\begin{array}{lllllllllllllllllllll}0.02 & 0.06 & \mathbf{0 . 0 3} & 0.05 & \mathbf{0 . 0 3} & 0.04 & 0.18 & 0.55 & \mathbf{0 . 6 4} & 0.32 & \mathbf{0 . 5 4} & 0.19 & 0.63 & 0.38 & \mathbf{0 . 6 8} & 0.02 & 0.07 & 0.10 & 0.20 & 0.46 & 0.27\end{array}$

$\begin{array}{lllllllllllllllllllll}371 & 408 & \mathbf{3 9 7} & 425 & \mathbf{4 0 5} & 451 & 539 & 674 & \mathbf{5 9 5} & 701 & \mathbf{6 1 5} & 715 & 897 & 1,120 & \mathbf{9 4 7} & 363 & 410 & 530 & 689 & 874 & 1,130\end{array}$

$\begin{array}{llllllllllllllllllllll}0.28 & 0.09 & \mathbf{0 . 0 3} & 0.18 & \mathbf{0 . 1 0} & 0.29 & 0.05 & 0.02 & \mathbf{0 . 0 0} & 0.03 & \mathbf{0 . 0 0} & 0.03 & 0.02 & 0.02 & \mathbf{0 . 0 0} & 0.08 & 0.04 & 0.02 & 0.02 & 0.02 & 0.02\end{array}$

$\begin{array}{lllllllllllllllllllll}0.48 & 0.17 & \mathbf{0 . 0 5} & 0.34 & \mathbf{0 . 2 2} & 0.55 & 0.11 & 0.04 & \mathbf{0 . 0 0} & 0.07 & \mathbf{0 . 0 1} & 0.09 & 0.06 & 0.04 & \mathbf{0 . 0 0} & 0.39 & 0.24 & 0.15 & 0.13 & 0.12 & 0.11\end{array}$

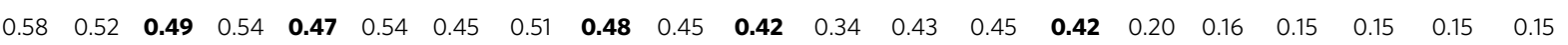

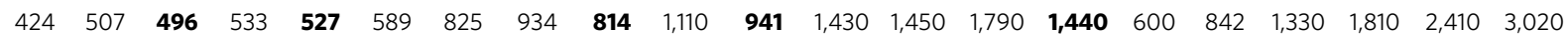

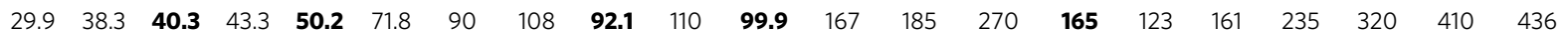

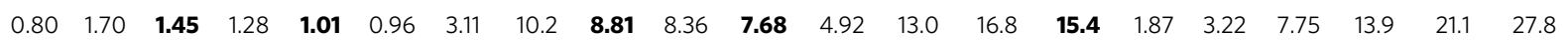

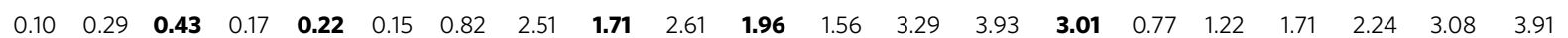

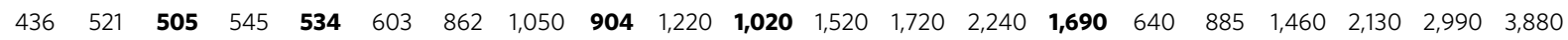

$\begin{array}{lllllllllllllllllllll}31.6 & 44.2 & \mathbf{4 3 . 6} & 47.1 & \mathbf{5 3 . 8} & 76.9 & 105 & 236 & \mathbf{1 6 2} & 193 & \mathbf{1 6 8} & 220 & 448 & 651 & \mathbf{3 5 8} & 138 & 183 & 278 & 424 & 621 & 758\end{array}$

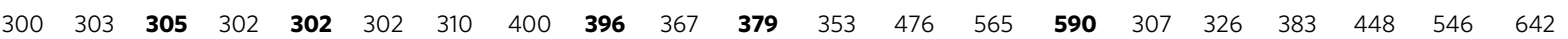

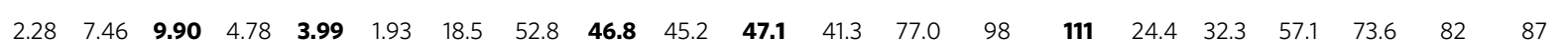

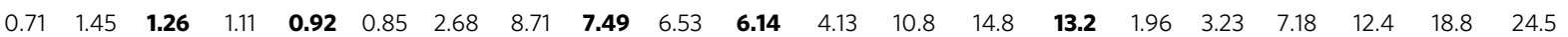

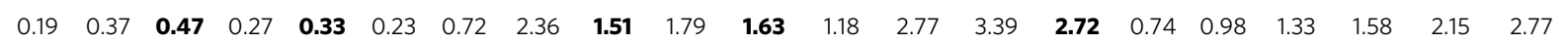

$\begin{array}{lllllllllllllllllllll}304 & 311 & \mathbf{3 1 4} & 308 & \mathbf{3 0 8} & 306 & 326 & 438 & \mathbf{4 3 1} & 396 & \mathbf{4 1 0} & 380 & 528 & 642 & \mathbf{6 4 9} & 324 & 349 & 428 & 519 & 625 & 741\end{array}$

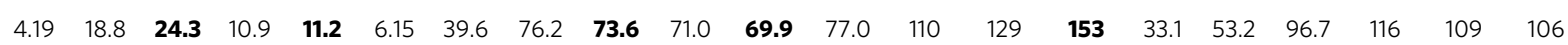

CC, carbonaceous chondrites; OC, ordinary chondrites

Initial matrix volume fraction varied from 70 to $33 \%$, producing post-impact matrix volume fraction similar to carbonaceous and ordinary chondrites, respectively. Impact velocities ranged from 0.75 to $3 \mathrm{~km} \mathrm{~s}^{-1}$. Bold entries show results for simulations with serpentine matrix and forsterite chondrules (approximating CM and CR scenarios). Other entries show results for forsterite matrix and forsterite chondrules.

In all simulations the initial temperature ( $T 0$ ) was $300 \mathrm{~K}$. Varying $T 0$ does not significantly affect $\delta T$ ( $4 \%$ lower at $T 0=600 \mathrm{~K}$ versus $T 0=300 \mathrm{~K}$ ).

${ }^{\star}$ Average pressure in the shock wave as it propagated through the sample region.

$\dagger$ Average temperature in the sample region after release from high pressure.

$\ddagger T$ (final) statistics use temperature of each tracer at time of release from high pressure.

$\$ P$ (peak) and $T$ (peak) statistics use extremes of pressure and temperature recorded by each tracer during passage of the shock wave. Note that the difference in average peak shock pressure between the chondrules and matrix is a consequence of the large strength difference between the components; the average peak longitudinal stress is the same in the chondrules and matrix (see Methods for more details).

\|The fraction of the matrix with $T$ (final) greater than the solidus temperature $(1,373 \mathrm{~K})$.

peak shock pressures $(P($ peak $))$ and post-shock temperatures (immediately after passage of the shock wave) ( $T$ (final)) across a computational mesh composed of a bimodal mixture of chondrules surrounded by a highly porous matrix using the iSALE hydrocode $\mathrm{e}^{17-19}$. Our approach complements and extends recent experimental work that tracked porosity in analogue materials ${ }^{8}$, allowing us to observe how pressure, temperature and porosity vary during an impact within and between chondritic components. Tracer particles recorded the peak- and post-shock state of the matrix and chondrule material, from which the bulk state was determined. We conducted 16 simulations (Table 1) that spanned a range in impact velocity $\left(0.75-3 \mathrm{~km} \mathrm{~s}^{-1}\right)$ and initial matrix volume fraction (70 and $33 \%)$ to simulate impacts that generated post-compaction matrix volume fractions consistent with CC-like objects and ordinary chondrite (OC)like objects, respectively. An ANEOS-derived equation of state table for forsterite ${ }^{20}$ was used to describe the thermodynamic response of the non-porous disks and the solid component of the 
a

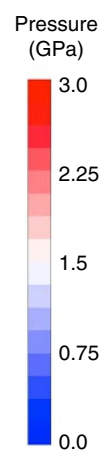

$v_{i}=1 \mathrm{~km} \mathrm{~s}^{-1} ; P($ bulk $)=1 \mathrm{GPa} ; T($ bulk $)=450 \mathrm{~K}$
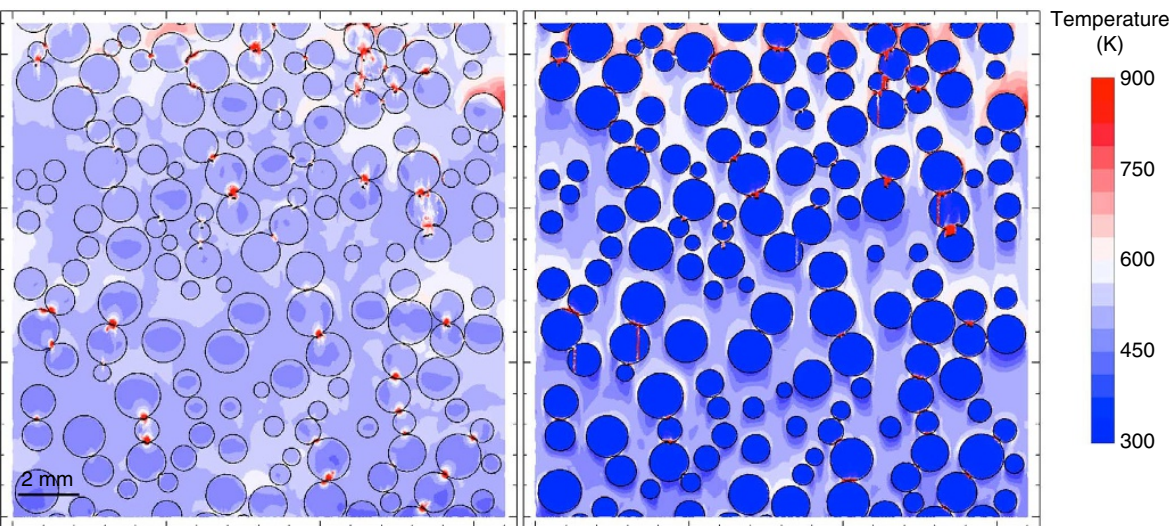

b

$v_{i}=2 \mathrm{~km} \mathrm{~s}^{-1} ; P($ bulk $)=3 \mathrm{GPa} ; T$ (bulk) $=650 \mathrm{~K}$
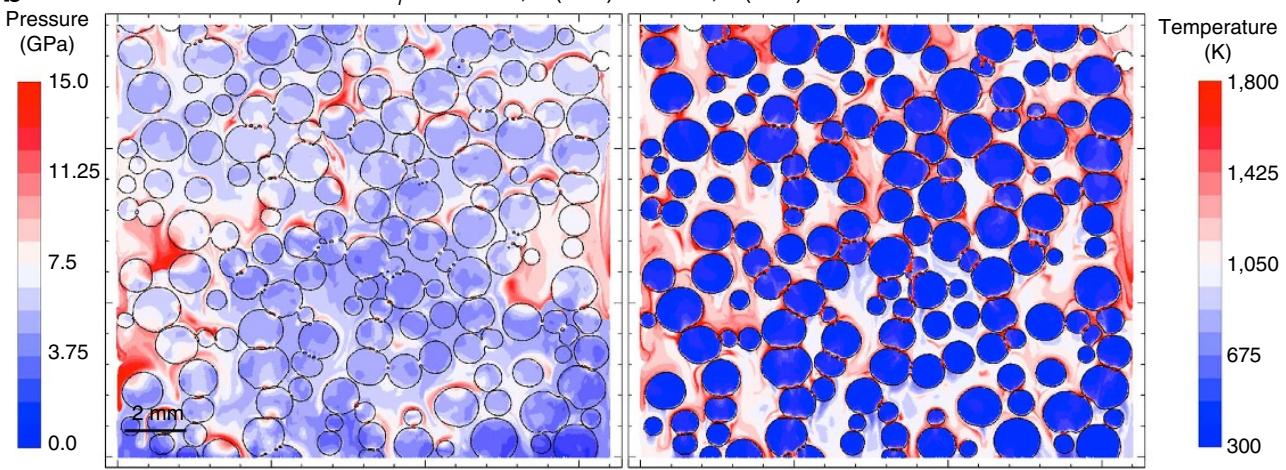

C
Pressure
(GPa)

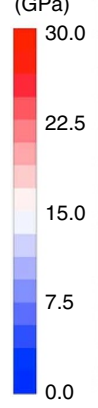

$v_{i}=3 \mathrm{~km} \mathrm{~s}^{-1} ; P($ bulk $)=8 \mathrm{GPa} ; T($ bulk $)=1,100 \mathrm{~K}$
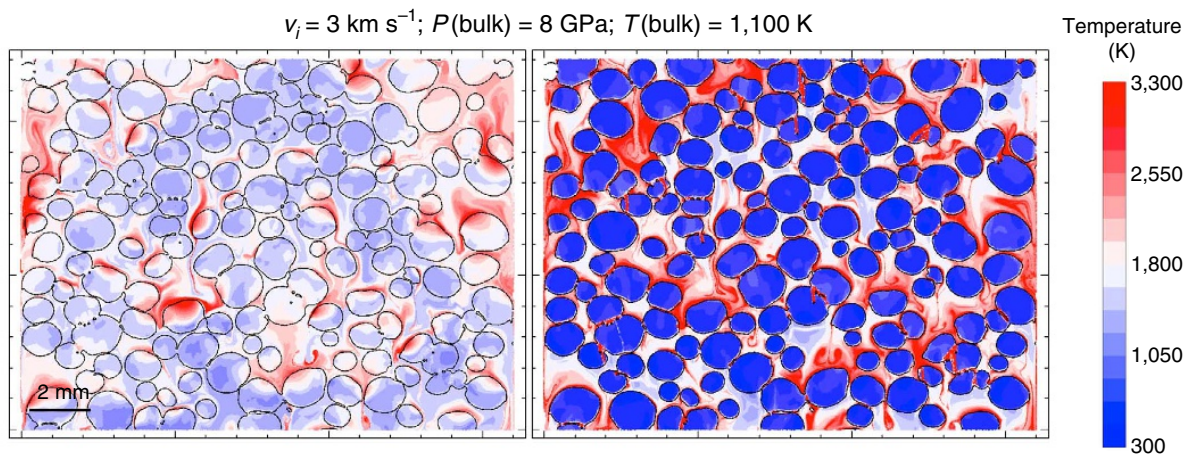

Figure 1 | Variation in peak-shock pressure and peak temperature in mesoscale simulations. In the examples shown, matrix is composed of forsterite, initial matrix volume fraction is $70 \%$, initial matrix porosity is $70 \%$ and starting temperature is $300 \mathrm{~K}$. (a) $1 \mathrm{~km} \mathrm{~s}^{-1}$ impact results in bulk shock pressure $\sim 1 \mathrm{GPa}$; (b) $2 \mathrm{~km} \mathrm{~s}^{-1}$ impact results in $P$ (bulk) $\sim 3 \mathrm{GPa}$; (c) $3 \mathrm{~km} \mathrm{~s}^{-1}$ impact results in $P$ (bulk) $\sim 7 \mathrm{GPa}$. The images illustrate the variation in $P$ (peak) and $T$ (peak) recorded by a representative portion of the sample after passage of the shock wave. Note the change in pressure and temperature scale between frames. The extreme variability in matrix PT over short lengthscales is apparent, indicated by swirling contours in matrix. Our simulations reproduce a variety of chondrule/matrix textures observed in the chondrite record (elongate or oriented chondrules; matrix 'flowing' between chondrules; indented chondrules). Chondrule indentation textures become apparent at $2 \mathrm{~km} \mathrm{~s}^{-1}\left(1.5 \mathrm{~km} \mathrm{~s}^{-1}\right.$ at lower matrix vol\% (for example, OCs)); internal chondrule $P($ peak ) $\sim 5 \mathrm{GPa}$.

matrix. We also conducted five simulations with serpentine matrix and forsterite chondrules (to approximate $\mathrm{CM}$ and $\mathrm{CR}$ chondrites), using ANEOS input parameters for serpentine ${ }^{21}$. Impact heating will lead to dehydration of hydrous phases, releasing water that may cause textural modifications, but computational limitations meant that we could not model this process explicitly. Compaction of porosity and material strength were modelled using methods described in earlier work ${ }^{18,19}$. We take $\phi_{\mathrm{mi}}=70 \%$ as a standard value in our simulations, with selected comparison runs at $\phi_{\mathrm{mi}}=60 \%$ and $\phi_{\mathrm{mi}}=80 \%$. Initial temperature was $300 \mathrm{~K}$ (an argument can be made that this should be higher, given that peak metamorphic temperature in
CV chondrites exceeded $600 \mathrm{~K}$, and compaction post-dated formation of secondary minerals). We explored the effect of varying $T$ (initial). For small $(100 \mathrm{~K})$ variations, temperature changes predicted by the models were independent of the starting temperature, for example, $100 \mathrm{~K}$ lower $T$ (initial) would reduce final temperatures by that amount.

Our simulations allow us to observe how pressure, temperature and porosity vary during an impact within and between chondritic components, observing detail down to the $100-\mu \mathrm{m}$ level (Fig. 1). Although low velocity impacts are expected to produce minor effects in $\mathrm{CCs}^{1,2}$, resolving at this finer 'mesoscale', we find that this is not the case. Predictably, 
matrix porosity is removed rapidly, decreasing from $40-50 \%$ at $0.75 \mathrm{~km} \mathrm{~s}^{-1}$ to $\sim 15 \%$ at $1.5 \mathrm{~km} \mathrm{~s}^{-1}$. But matrix and chondrules experience very different conditions, particularly with respect to temperature, in all scenarios (Fig. 1). A $2.0-\mathrm{km} \mathrm{s}^{-1}$ event into a $70 \%$ forsterite matrix precursor (Fig. 1b) with $\phi_{\mathrm{mi}}=70 \%$ generated chondrule $P$ (peak) of $6.5 \pm 1.8 \mathrm{GPa}$ : barely detectable in a typical petrographic study ${ }^{1,2}$. However, matrix experiences $T$ (final) of $1,100 \mathrm{~K}$ while chondrules are virtually unheated $(\Delta T$ (final) $\sim 70 \mathrm{~K})$. We also observe significant heterogeneity in PT conditions over short $(100 \mu \mathrm{m})$ lengthscales. Taking the same example $\left(2.0 \mathrm{~km} \mathrm{~s}^{-1}\right.$ and $70 \%$ initial matrix volume fraction: Fig. 1b), we observe a 7-18-GPa range of $P$ (peak) over $100 \mu \mathrm{m}$ in matrix (average matrix $P$ (peak) is $8.4 \pm 2.6 \mathrm{GPa}$ in this simulation), and a $700-2,000-\mathrm{K}$ variation in matrix $T$ (final) over similar lengthscales (average matrix $T$ (final) is $1,100 \mathrm{~K}$ ). Chondrule deformation (and flattening) is apparent at velocities of $2 \mathrm{~km} \mathrm{~s}^{-1}$ and higher. The assumed Hugoniot Elastic Limit for the chondrules $(\sim 5 \mathrm{GPa})$ is exceeded in these higher-velocity simulations, that is, the pressure is so high that they are deformed in a plastic, rather than in a brittle manner, despite the high strain rate. We also note that chondrule edges experience higher shock than chondrule interiors. This occurs where chondrules impact and indent one another (leading to local $P$ (peak) spikes up to $\sim 12 \mathrm{GPa}$ in the $2-\mathrm{km} \mathrm{s}^{-1}$ simulation), but it is also a general feature, as matrix compacts around chondrules. Although average chondrule $P$ (peak) is $6.5 \mathrm{GPa}$ at $2 \mathrm{~km} \mathrm{~s}^{-1}$, interiors only see 4-5 GPa, while chondrule edges range up to $8 \mathrm{GPa}$ (it is probable that the real value is higher, but spatial resolution is limited by computational costs). Finally, with regard to the serpentine matrix scenarios, at low shock pressure there is little difference in matrix $T$ (final) between serpentine and forsterite. In simulations $\geq 2 \mathrm{kms}^{-1}$ temperature is buffered by phase changes in serpentine, reducing matrix temperatures in these higher-shock scenarios. However, even here the same general features remain: high matrix heating alongside 'cold' chondrules, and significant PT heterogeneity over short lengthscales.

Previous studies examined shock effects in porous targets, or non-porous targets, but not mixtures. It is known that impacts into porous materials result in much more heating than impacts into non-porous materials, as shock energy is expended in collapsing pores ${ }^{3,4}$. Impacts into a uniformly porous asteroid will compact that material, heating a large volume around the crater that then cools and equilibrates over timescales up to $10-100 \mathrm{Myr}^{11}$. Primitive chondrites are compacted, but are texturally and compositionally unequilibrated. Our data indicate that impact-induced compaction in chondrites occurred without generating macroscopic thermal metamorphic textures, or chemical equilibration, because of the juxtaposition of two materials with vastly different initial porosities and bulk properties. In the chondrite scenario, chondrules act as a heat sink. Matrix is heated rapidly to 100's of degrees above ambient, but because of the proximity to 'cold' chondrules, it cools rapidly. Rather than slow cooling of a large volume ${ }^{11}$, we see sub-mm heterogeneities that equilibrate in $<10$ s. So unless bulk $T$ (final) exceeds temperatures typical of CC peak metamorphism (a condition we observe only in scenarios with impact velocity $\left.>2-2.5 \mathrm{~km} \mathrm{~s}^{-1}\right)$, macroscopic effects will not be apparent.

Electron microscopy. Although the effect of shock on finegrained crystalline solids has been investigated ${ }^{22}$, there are few detailed transmission electron microscopy (TEM) studies of shock metamorphism in the meteorite matrix ${ }^{9}$. And while petrographic studies of experimentally shocked chondrites are informative ${ }^{23}$, the 'unshocked' starting point in this work is a meteorite that has already experienced significant compaction.
Given the limited literature, we conducted our own coordinated field emission (FE)-electron probe microanalysis (EPMA), FE-scanning electron microscope (SEM) and focused ion beam (FIB)/TEM study of a CR2 (GRA 06100), focussing specifically on how impact-induced processes may have affected sub- $\mu \mathrm{m}$ materials in this rock. We devised a novel methodology that used temperature-related crystallographic transitions to quantify matrix temperatures at fine scale. To select regions to extract FIB sections, we first built a map based on FE-SEM back-scattered electron and energy-dispersive spectrometry (EDS). The results of the FIB analysis guided us in interpreting detailed mineralogy from the original map. This combined approach enabled us to draw the first detailed isotherm map for a chondrite, in this case GRA 06100 (Fig. 2) (a follow-up study ${ }^{10}$ in which an additional eight FIB sections were extracted found that the mineralogy was consistent with expectations at all the new sites).

No evidence of heating was found in chondrule olivine and pyroxenes. GRA 06100 matrix consists of sub-micron, diffuse masses that have compositions ranging from low-total, $\mathrm{Mg}$, Al-bearing, saponite to ferroan serpentine under a $10-\mu \mathrm{m} \mathrm{EPMA}$ beam. The porosity and mineralogy of GRA 06100 matrix is variable, containing deformed $\mathrm{SiO}_{2}$ (grains contain nanocrystalline and amorphous regions), Fe-silicide, nanophase fayalitic olivine and ferrosilite, non-stoichiometric ferromagnesiosilica grains, as well as amorphous silicates and phyllosilicates with variable amounts of Al. Although Fe-rich serpentine is the most common matrix phyllosilicate, the smectite aliettite was identified in several regions of the matrix, using a combination of TEM/ EDS and crystallography. Aliettite undergoes four endothermic transitions $\left(150,220,605 \text { and } 905^{\circ} \mathrm{C}\right)^{24}$, which are of use in determining the minimum temperature reached in a region of matrix. Aliettite recording $220^{\circ} \mathrm{C}$ was found in close proximity to aliettite recording 605 and $905^{\circ} \mathrm{C}$.

Near-end member ankerite was found at the edge of a large metal nodule, located $<20 \mu \mathrm{m}$ from unheated chondrule olivines. Ankerite of this composition is predicted to occur at $T>827^{\circ} \mathrm{C}$ (ref. 25). Also $<20 \mu \mathrm{m}$ from unheated chondrule olivines, the high-temperature silicate-sulfate-phosphate scorzalite-lazulite was found. The observed compositions in the scorzalite-lazulite solid solution correspond to $T>485^{\circ} \mathrm{C}$ (ref. 26). Finally, pentlandite, the most common sulphide in $\mathrm{CR}$ chondrites, is absent from GRA 06100. Where sulphides are present, they consist of pyrrhotite and partly oxidized Fe-sulfides sub-domains. Thermal decomposition of pentlandite occurring in opaque assemblages in GRA 06100 occurs at $T>600^{\circ} \mathrm{C}$ (ref. 27).

On the basis of the distribution of different minerals of selected areas of the thin section, we produced a thermal contour map (Fig. 2b). We estimate that $\sim 46 \%$ of the section shows no evidence of heating, $\sim 12 \%$ is consistent with $493 \mathrm{~K} \leq T \leq 873 \mathrm{~K}$, $\sim 28 \% \quad 873 \mathrm{~K} \leq T \leq 1,023 \mathrm{~K}, \quad \sim 9 \% \quad 1,023 \mathrm{~K} \leq T \leq 1,178 \mathrm{~K}$ and $\sim 5 \%$ records temperatures $\geq 1,178 \mathrm{~K}$. However, the great diversity of mineral assemblages identified in matrix indicates that more FIB sections are needed to fully constrain the temperature regimes recorded by fine-grained material. Note: although phase transition temperatures are defined at low pressure, the comparison with model results is valid as we are comparing to post-shock/pre-equilibration temperatures (that is, low pressure after shock passage but before thermal equilibration between chondrules and matrix).

Macroscale simulations. In addition to the mesoscale simulations, a suite of 'macroscale' planetesimal collision simulations were performed (for example, Fig. 3). Tracer particles were used to track planetesimal material exposed to different bulk postshock temperatures for comparison with the mesoscale 


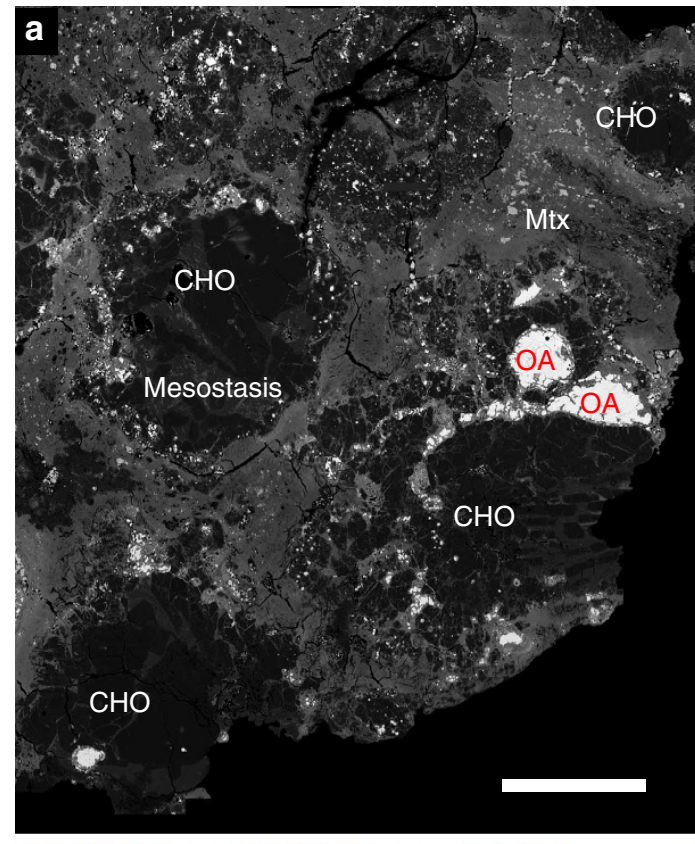

Temp (K)
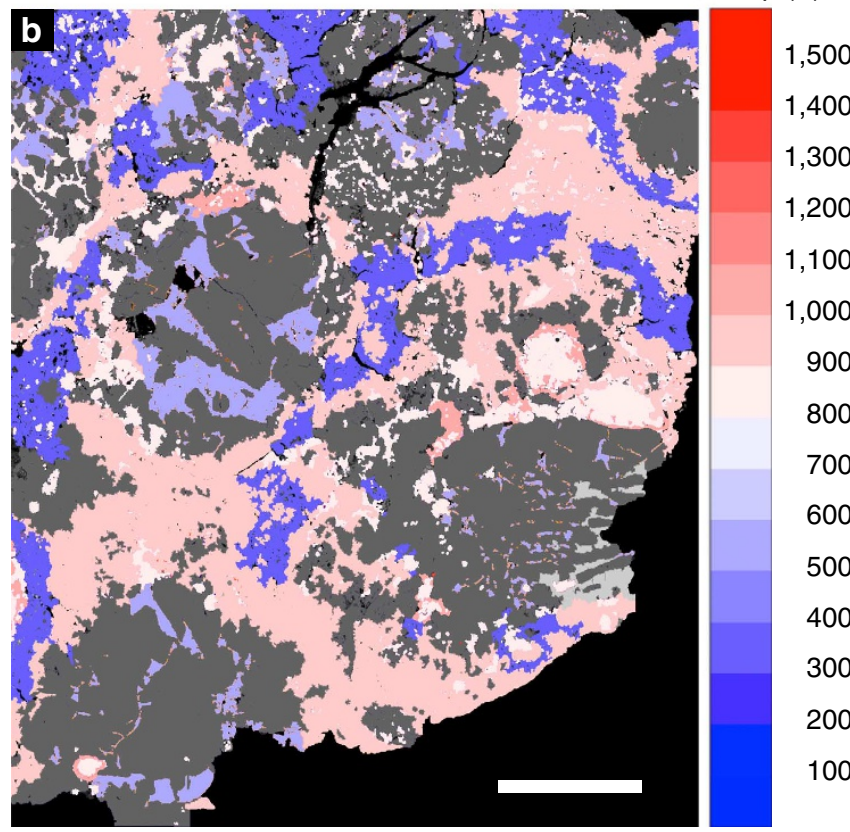

Figure 2 | Back-scattered electron (BSE) and temperature images of CR2 chondrite GRA 06100. (a) BSE image showing chondrules (CHO), mesostasis, opaque assemblages (OA) and matrix (Mtx). (b) Temperature map of the same area. Scale bar, $0.5 \mathrm{~mm}$. Chondrule olivines (gray-scale regions) show no evidence of heating or shock. Temperatures were estimated based on the crystallographic transitions of aliettite $(423,493$, 878 and $1,178 \mathrm{~K})^{24}$; presence of near-end member ankerite $(T>1,100 \mathrm{~K})^{25}$; thermal decomposition of pentlandite $(T>873 \mathrm{~K})^{27}$; scorzalite-lazulite solid solution $(T>758 \mathrm{~K})^{26}$

simulation results and meteoritic constraints (Fig. 3). The mass of material in the range $400 \mathrm{~K}<$ bulk $T$ (final) $<700 \mathrm{~K}$ is shown in blue; material that experienced bulk $T$ (final) $>700 \mathrm{~K}$ is shown in green $(700-1,000 \mathrm{~K})$ and red $(>1,000 \mathrm{~K})$. Figure 4 shows the total mass of material heated to different final bulk temperatures from an example suite of collisions (1:1,000 impactor-to-target mass ratio; $50 \%$ initial porosity in impactor and target) at various impact speeds using the same colour coding. All macroscale simulations assumed head-on collisions between planetesimals,

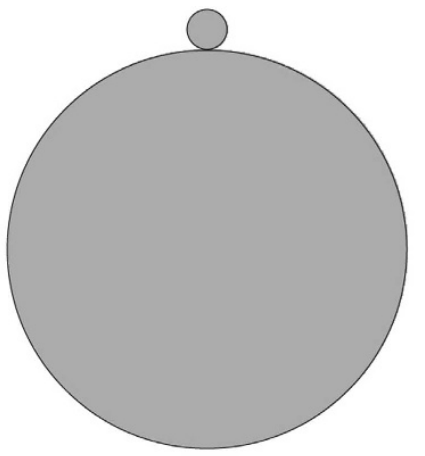

$0 \mathrm{~s}$

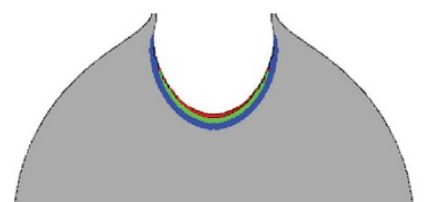

$250 \mathrm{~s}$
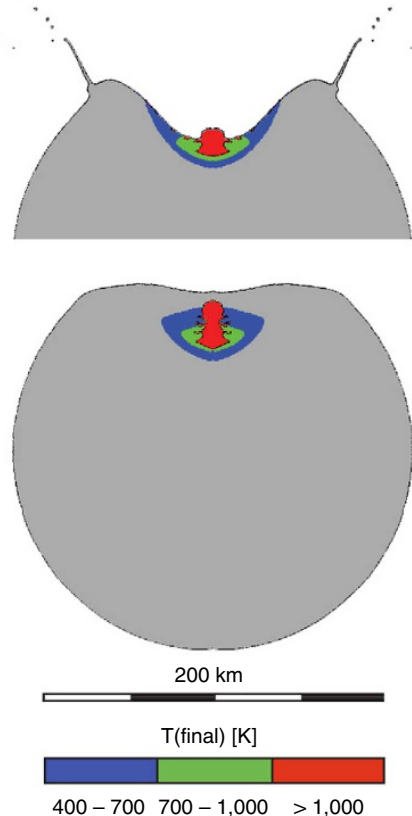

$1,500 \mathrm{~s}$

$8,500 \mathrm{~s}$

Figure 3 | Translating mesoscale observations into asteroid-scale impacts. Macroscale hydrocode modelling of impacts between porous planetsimals. In an impact on a real planetesimal, the shock wave attenuates rapidly with distance, but the peak shock PT conditions are similar to the planar impact scenario. Macroscale models consider impact velocities from 1 to $10 \mathrm{~km} \mathrm{~s}^{-1}$ for both OC-like (20\% initial bulk porosity) and CC-like (50\% initial bulk porosity) objects; assume vertical impact; and with target planetesimals of 200-500 km diameter, and impacting planetesimals of $10-150 \mathrm{~km}$ in diameter. This example involves CC-like objects impacting at $4 \mathrm{~km} \mathrm{~s}^{-1}$. The blue region logs tracers at $400 \mathrm{~K}<$ bulk $T$ (final) $<700 \mathrm{~K}$ : the range consistent with meteorite data. Of the material that is compacted to meteorite-like porosities, we assume (conservatively) that if $>20 \%$ experienced bulk $T$ (final) $>700 \mathrm{~K}$ (shown here in green $(700-1,000 \mathrm{~K})$ and red $(>1,000 \mathrm{~K})$ ) it would be observed in the meteorite record. This corresponds to velocities exceeding $3 \mathrm{~km} \mathrm{~s}^{-1}$ in modelled impacts (Fig. 4). Similar velocities are derived from OC-like simulations.

enforced by the $2 \mathrm{D}$ axial symmetry of the numerical model. In reality, the most common impact angle is $45^{\circ}$ to the target plane. Numerical simulations of oblique impacts on planar surfaces suggest that the volume of material heated to a given temperature scales with vertical component of impact velocity in the same way as for vertical impacts ${ }^{28}$. Hence, in the context of 
a

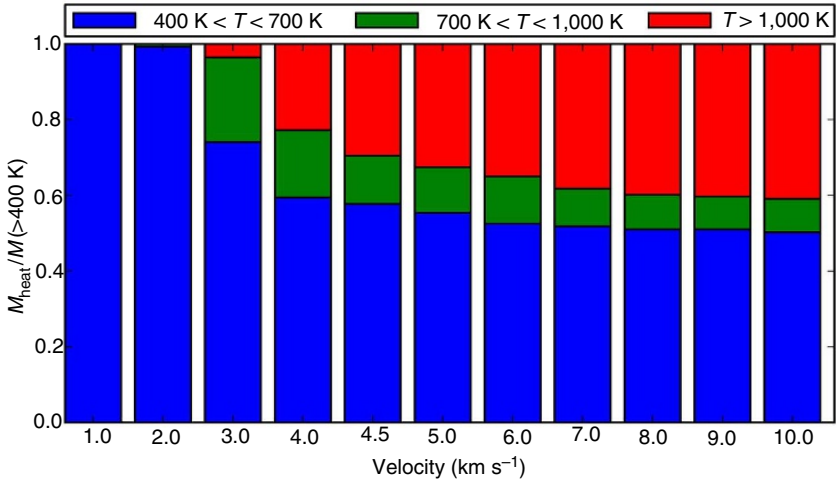

b

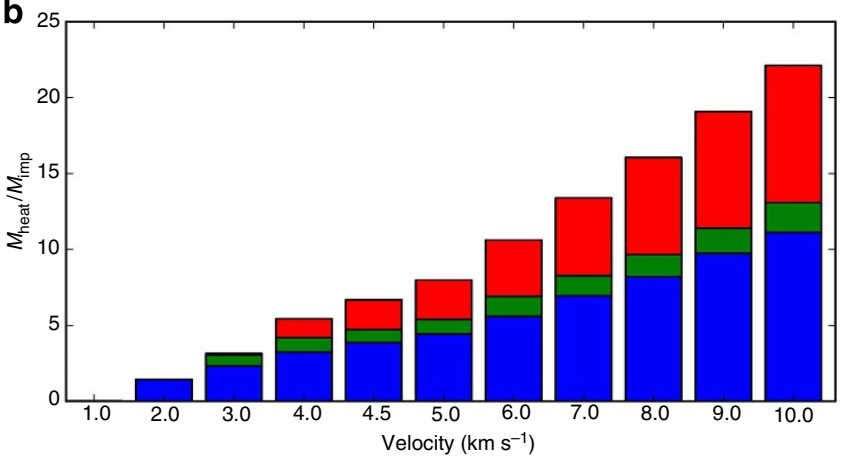

Figure 4 | Fraction of material heated to varying temperature in macroscale collisions. Results are shown for head-on collisions between two planetesimals with mass ratios of 1:1,000 and with initial porosities of $50 \%$ (CC-like simulation). Equivalent simulations were performed for OC-like (20\% initial bulk porosity) objects, and for a range of other target and impactor diameters. Results are normalized by both the impactor mass (b) and the mass of material heated by $100 \mathrm{~K}$, which corresponds to a bulk porosity reduction of $\sim 10 \%$ (a). The fraction of material heated above $700 \mathrm{~K}$ exceeds 0.2 at a vertical impact velocity of $\sim 3 \mathrm{~km} \mathrm{~s}^{-1}$. Similar velocities are derived from $\mathrm{OC}$-like simulations. The $2 \mathrm{D}$ axial symmetry of the numerical model enforces head-on collisions between planetesimals in the macroscale simulations. In reality, the average impact angle is $45^{\circ}$ to the target plane. The volume of material heated to a given temperature also scales with a vertical component of impact velocity in the same way as for vertical impacts ${ }^{28}$. Thus, a $3-\mathrm{km} \mathrm{s}^{-1}$ vertical impact is approximately equivalent to a $45^{\circ}$ impact at $3 / \sin (45)=4.25 \mathrm{~km} \mathrm{~s}^{-1}$.

Fig. 4, a $3-\mathrm{km} \mathrm{s}^{-1}$ vertical impact is approximately equivalent to a $45^{\circ}$ impact at $3 / \sin (45)=4.25 \mathrm{~km} \mathrm{~s}^{-1}$.

\section{Discussion}

Our observations have a number of implications for understanding the chondrite record: lithification of primordial materials may have been a natural outcome of transient heating of matrices, driven by collisional compaction. Hydrothermal alteration has been suggested as a mechanism for meteorite lithification, but some primitive meteorites have escaped this process and yet are consolidated rocks. Lithostatic pressure was minimal $^{16}$, and towards the surface-where primitive chondrites are expected to originate-it would have been even lower. Yet chondrite lithification was efficient even at fine scales: matrices are lithified. Although collisional compression has been proposed as a mechanism ${ }^{16}$, impact shock has generally been excluded as few CCs show macroscopic shock indicators. But our data indicate that this is not a valid constraint when considering the response of matrix to shock. In addition, experiments examining

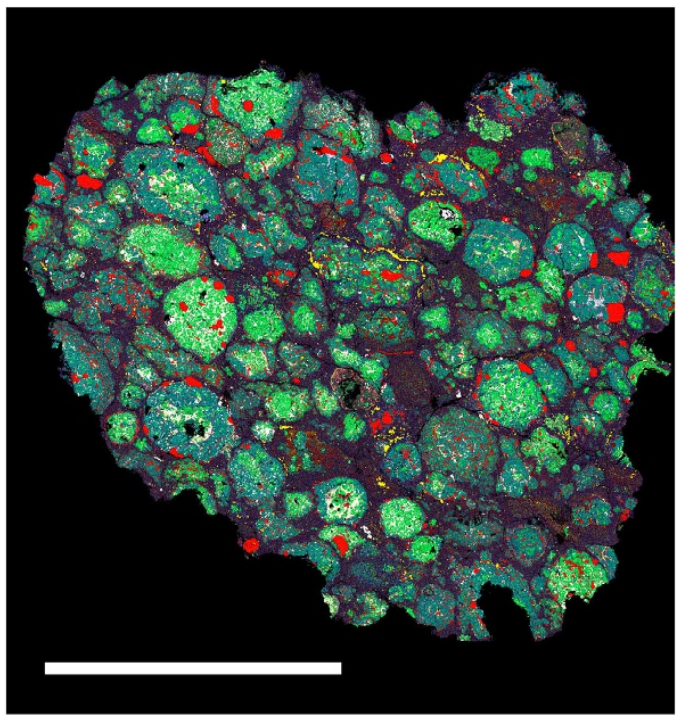

Figure 5 | Chondrule textures in the CR2 chondrite Renazzo. Flattened and indented chondrules highlighted in an element map of Renazzo (green:Mgl yellow:Ca; white:Al; red:Fe; blue:Si); textures similar to the $2-\mathrm{km} \mathrm{s}^{-1}$ simulation shown in Fig. 1b. Scale bar $6 \mathrm{~mm}$.

the sintering behaviour of olivine have found that it is favoured where a fine-grained (micron-scale) aggregate is rapidly heated to high $T^{29}$ : a situation identical to that encountered by highporosity matrix during impact-induced compaction.

Our results have implications for chondrite palaeomagnetism. Magnetic fields may be recorded at the grain scale without raising the bulk temperature above the Curie point; and this can occur without generating obvious macroscopic shock metamorphic textures (that is, it may have affected meteorites that have been classified as low shock). It is known that an ambient magnetic field at the time of impact may be amplified or even produced by the impact itself ${ }^{30}$. Large impact-generated fields are possible ${ }^{31}$. But impact-generated fields have not been considered a significant factor in the palaeomagnetic record of primitive chondrites for two principal reasons. It was thought that shock pressures $>40 \mathrm{GPa}^{30}$ were required to generate temperatures high enough to impart a thermoremanent magnetization (TRM). In these highly energetic events post-impact cooling times would be long (perhaps $10^{5}-10^{7} \mathrm{Myr}$ ). But impact-generated fields are brief ( $<$ mins). An additional reason is the absence of macroscopic shock textures in most $\mathrm{CCs}^{30}$. Our work indicates that these arguments should be re-examined. We show that it is necessary to model PT evolution at the meteorite scale, in order to constrain laboratory measurements at the meteorite scale. The absence of shock textures in chondrules does not inform our understanding of matrix PT history. The bulk rock will respond as expected in the literature, but on a finer scale, matrix will experience rapid heating and cooling, prior to equilibrating to bulk $T$ (final) in $<10$ 's of seconds. If the principal magnetic carrier phase was hosted in matrix (as is often the case), then the palaeomagnetic record may well have been modified during compaction. Equally, a matrix phase could record an impactgenerated field with a duration of 10's of seconds to minutes. A rock could acquire a TRM during rapid cooling of matrix following impact-induced compaction.

Model results showing PT heterogeneity at fine scale and high transient matrix temperatures suggest a unified explanation for anomalous features of chondrite petrography and texture that previously have required complex mechanisms (involving accretion, disruption and dispersal, heating or shock processing in the 
Table 2 | Material parameters used in numerical simulations.

\begin{tabular}{|c|c|c|c|c|}
\hline Compaction rate ${ }^{\star}$ & NA & 0.98 & 0.98 & 0.98 \\
\hline Poisson ratio (solid component) ${ }^{\dagger}$ & 0.23 & 0.23 & 0.23 & 0.23 \\
\hline Intact cohesion ${ }^{\dagger}(\mathrm{MPa})$ & 1,000 & 0.1 & 0.1 & 10 \\
\hline Intact friction coefficient ${ }^{\dagger}$ & 1.2 & 1.2 & 1.2 & 1.2 \\
\hline Intact strength $\operatorname{limit}^{\dagger}(\mathrm{GPa})$ & 3.5 & 0.035 & 0.035 & 3.5 \\
\hline Damaged strength $\operatorname{limit}^{\dagger}(\mathrm{GPa})$ & 3.5 & 0.035 & 0.035 & 3.5 \\
\hline Melt temperature ${ }^{3}$ (zero pressure) (K) & 1,373 & 1,373 & 1,098 & 1,373 \\
\hline Simon approximation constant ${ }^{\ddagger}(\mathrm{GPa})$ & 1.52 & 1.52 & 1.52 & 1.52 \\
\hline Simon approximation exponent ${ }^{\ddagger}$ & 4.05 & 4.05 & 4.05 & 4.05 \\
\hline Thermal softening parameter ${ }^{\dagger}$ & 1.2 & 1.2 & 1.2 & 1.2 \\
\hline
\end{tabular}

a

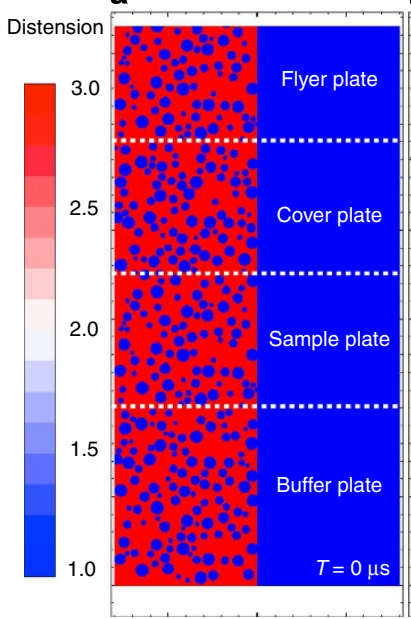

b

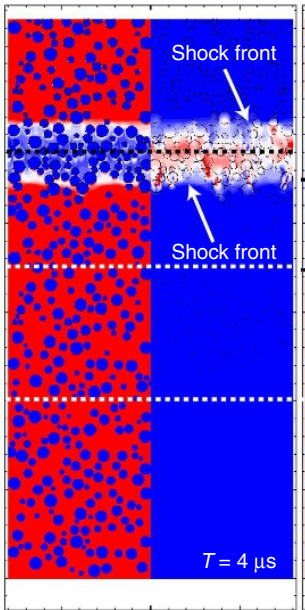

c

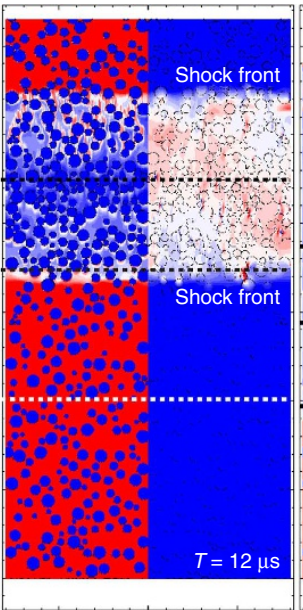

d

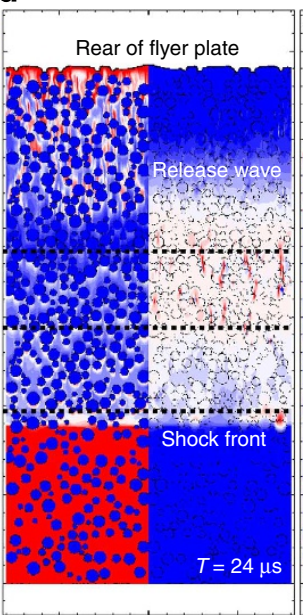

e

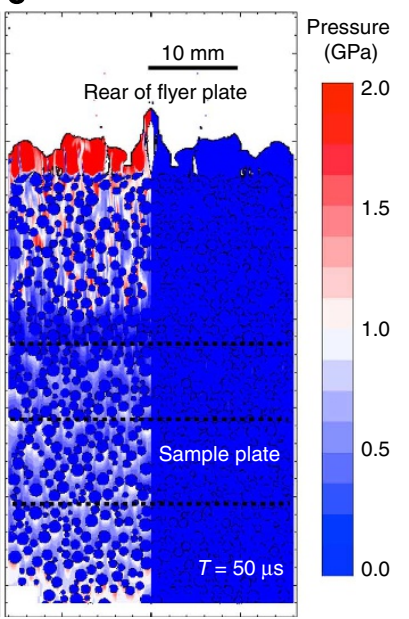

Figure 6 | Time sequence of a typical mesoscale impact simulation. The nominally planar shock wave is shown propagating through a bimodal mixture of explicitly resolved non-porous chondrules surrounded by a high-porosity matrix, with snapshots showing the initial state (a), at $4 \mu \mathrm{s}$ (b), $12 \mu \mathrm{s}$ (c), $24 \mu \mathrm{s}$ (d), and ending at $50 \mu \mathrm{s}$ (e). Scale bar, $10 \mathrm{~mm}$. Each time snapshot is divided into two panels, with colour-scales denoting distension (1/(1- porosity)) (high initially in matrix), and instantaneous longitudinal stress ( 0 initial throughout the mixture). Upon impact, shockwaves are generated at the flyersample interface and propagate both down into the sample and up into the flyer plate, compacting the matrix. In this example, after $\sim 24 \mu$ s the shock wave in the sample has reached the sample/buffer plate interface; by this time the shock in the flyer plate has reflected off the rear of the flyer plate as a release wave that propagates back through the flyer, sample and buffer plates. By $50 \mu$ s the release wave has left the computational domain and the post-shock state of the sample may be recorded. The variation in peak pressure, peak- and post-shock temperature experienced by both the chondrules and matrix was recorded for subsequent analysis as was the reduction in porosity in the matrix.

nebula, followed by re-accretion and (possibly) brecciation ${ }^{32,33}$ ). Features such as shock-induced mobilization of sulphides in (apparently) 'unshocked' $\mathrm{OCs}^{32}$, pyroxene polymorphs in CC matrices that require a high-temperature origin $(>1,300 \mathrm{~K})$ followed by very rapid cooling 33,34 and matrix olivines showing dislocations indicative of significant shock deformation with adjacent chondrule olivines unaffected ${ }^{9,35}$, are entirely consistent with our observations. In addition, flattened, oriented or indented chondrules are a feature of many primitive meteorites, even those characterized as very weakly shocked (for example, Renazzo, S2; Fig. 5). In our modelling these chondrule textures become apparent at $2 \mathrm{kms}^{-1}$ (Fig. 1b). Chondrule $P$ (peak) in the forsterite simulation is $6.5 \mathrm{GPa} \quad(6.1 \mathrm{GPa}$ with serpentine matrix): according to macroscopic shock indicators in chondrules, this is consistent with shock level S2 (very weakly shocked). CR chondrites are among the most pristine CCs: we might expect them to preserve the most complete record of early compaction. The detailed isotherm map generated from our TEM study of CR chondrite matrix revealed the degree of heterogeneity in GRA 06100. Although (as expected in a C2 chondrite) a large fraction of matrix shows little evidence of heating, mineral indicators in $>50 \%$ of matrix are consistent with temperatures $>900 \mathrm{~K}$, and we are able to define areas that experienced $T>1,180 \mathrm{~K}$ (ref. 24). We observe $1,000 \mathrm{~K}$ heterogeneity on $100 \mu \mathrm{m}$ lengthscales. In a highly unequilibrated meteorite this required rapid heating and cooling. Chondrules show no evidence of shock metamorphic textures. These observations are all consistent with our model predictions. In the case of GRA 06100 , they are a match to a $\sim 1.5-\mathrm{km} \mathrm{s}^{-1}$ scenario with $70 \%$ initial matrix by volume. Finally, a specific model prediction- 

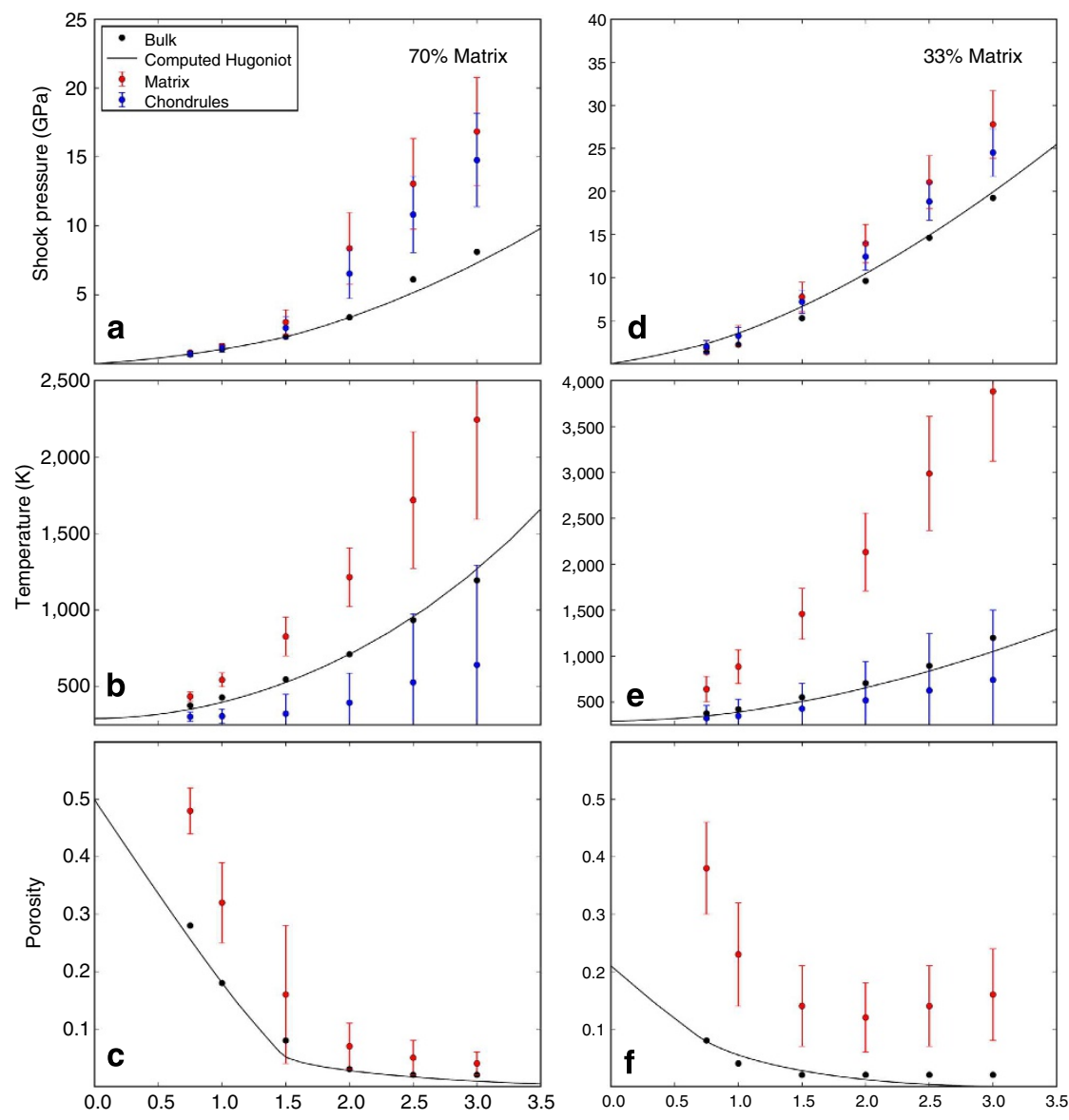

Impact velocity $\left(\mathrm{km} \mathrm{s}^{-1}\right)$

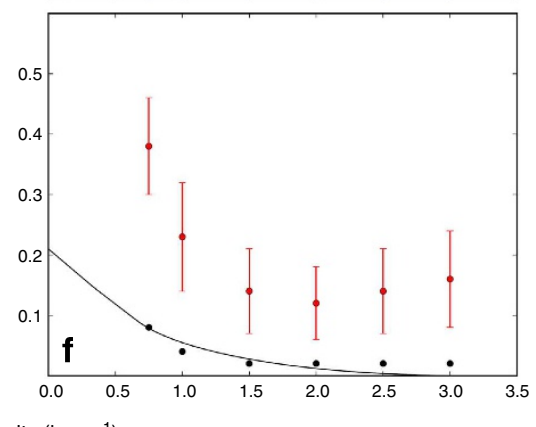

Figure 7 | Pressure, temperature and porosity in mesoscale simulations. Shock pressure (a,d), post-shock temperature (b,e) and post-shock porosity $(\mathbf{c}, \mathbf{f})$ as a function of impact velocity for bimodal chondrule/matrix mixtures with matrix volume fraction $70 \%$ (a-c) and $33 \%$ (d-f). Black symbols show bulk values of the state quantity, averaged over the entire sample region; red and blue symbols (and error bars) show mean (and s.d.) of the state variable in the matrix and chondrule fraction, respectively. Apart from the peak-shock pressures in the matrix and chondrules, which represent the maximum pressure recorded by the material at any time, all variables are measured at a given instant in time either during the shock passage (bulk pressure) or after release (final temperature and porosity). As a result, while the bulk porosity and final temperature values represent volume-weighted averages of the porosity and temperature of the matrix and chondrules components, the bulk shock pressure values are substantially less than the peak-shock pressures recorded by matrix and chondrules. Solid line shows a Hugoniot curve for the bulk porous material (0.49 for $70 \%$ matrix; 0.23 for $33 \%$ matrix) computed using the epsilon-alpha porous compaction model.

local melting of matrix in the most compacted primitive chondrites-has also been observed. Leoville is a reduced CV chondrite with porosity of $2 \%$ (ref. 14). A suite of observations, including highly variable shock effects on $100-\mu \mathrm{m}$ scales, and local matrix melting, was explained by a combination of hot accretion and impact ${ }^{36}$. Our modelling suggests a simpler, unified explanation.

The apparent connection between shock level (increasing from CCs, through unequilibrated ordinary chondrites, to equilibrated ordinary chondrites (EOCs)) and asteroid metamorphism ${ }^{1,2}$ has prompted some to suggest that asteroid thermal metamorphism was a result of large impacts ${ }^{37}$. Mesoscale simulations showing PT variability with matrix fraction provide an alternative explanation. At an impact velocity of $2 \mathrm{~km} \mathrm{~s}^{-1}$, chondrule interiors in a CC-like (high matrix fraction) precursor experience $P$ (peak) of $\sim 5 \mathrm{GPa}$; an OC-like (low matrix fraction) precursor $\sim 12 \mathrm{GPa}$; and a non-porous dunite (EOC approximation) $\sim 24 \mathrm{GPa}$. In other words, shock level varies from S1-S4 depending on matrix fraction for the same impact velocity. Shock level is derived from observation of textures in chondrule olivines. There is a widely held view that varying shock level informs us about varying intensity of asteroidal collisions. But our data suggest that the way we use macroscopic textures to infer shock level can bias our interpretation. It is not required that varying impact energy caused the differences in shock level between the chondrite groups. Rather, varying matrix proportion in chondrite targets controlled the level of shock metamorphism recorded by the chondrules within them.

Both in terms of locating impact-induced compaction within the meteorite record, and exploring its significance with respect to the dynamical evolution of planetesimals, it is useful to constrain the timing of compaction. Chondrule formation defines an upper limit on chondrite accretion times. Chondrites could not have accreted prior to the formation of the youngest chondrules that they contain. In addition, it is apparent from $\mathrm{CV}^{7,15}$ and $\mathrm{CR}$ (this study) data that compaction occurred after aqueous alteration. Recent ${ }^{207} \mathrm{~Pb}-{ }^{206} \mathrm{~Pb}$ ages for $\mathrm{CV}$ calcium-aluminium inclusions (CAIs) (the assumed starting point for solar system formation) are $4567.18 \pm 0.50 \mathrm{Myr}^{38}$ and $4567.30 \pm 0.16 \mathrm{Myr}^{39}$. Using current ${ }^{238} \mathrm{U} /{ }^{235} \mathrm{U}$ values ${ }^{38}$ to correct published chondrule ages drives chondrule $\mathrm{Pb}$-isotopic ages $0.95 \mathrm{Myr}$ younger: $4,564.5 \pm 0.50 \mathrm{Myr}$ for a multichondrule fraction from Allende ${ }^{40}, 4,563.23 \pm 0.66 \mathrm{Myr}$ for a multichondrule fraction from a $\mathrm{CR}^{41}$ (we can anticipate that individual chondrules within that fraction would have formed 
later than the $4,563.23 \pm 0.66 \mathrm{Myr}$ average). Analysis of two individual Allende chondrules generated ages of $4,567.32 \pm 0.42 \mathrm{Myr}$ and 4,566.24 $\pm 0.63 \mathrm{Myr}^{39}$. Relative ${ }^{26} \mathrm{Al} /{ }^{27} \mathrm{Al}$ chondrule ages agree, indicating that $\mathrm{CC}$ chondrules were still forming at $4.3 \mathrm{Myr}$ after $\mathrm{CAI}^{42}$. Recent ${ }^{53} \mathrm{Mn}-{ }^{53} \mathrm{Cr}$ ages for secondary fayalite in CVs are $3.9(+1.0 /-0.9) \mathrm{Myr}^{43}$. Together, these data allow us to say that CC compaction did not occur earlier than 4-5 Myr after CAI. The OC data are more variable. Three chondrules from NWA 5697 have ages that range from $4,566.67 \pm 0.43$ to $4,564.71 \pm 0.30 \mathrm{Myr}^{39} .{ }^{26} \mathrm{Al} /{ }^{27} \mathrm{Al}$ suggests that the youngest $\mathrm{OC}$ chondrules date to $\sim 3.5 \mathrm{Myr}$ after $\mathrm{CAI}^{42}$. And ${ }^{53} \mathrm{Mn}-{ }^{53} \mathrm{Cr}$ for secondary fayalite from an OC gave $2.4(+1.8 /$ $-1.3) \mathrm{Myr}^{44}$ after CAI. On balance, these data suggest an upper bound for compaction of OC planetesimals at $\sim 3.5 \mathrm{Myr}$ after CAI. Considering the chronometry data, our mesoscale simulations allow us to define a 'speed limit' constraint on the major compressive impact event(s) affecting CC planetesimals after 4-5 Myr, and OC planetesimals after $\sim 3.5 \mathrm{Myr}$.

A spectrum of meteorite data (porosities, meteorite fabrics, petrography and local PT heterogeneity) are consistent with compaction from high $\phi_{\mathrm{mi}}$ at velocities in the range $0.75-$ $2 \mathrm{~km} \mathrm{~s}^{-1}$ (bulk $T$ (final) $\sim 400-700 \mathrm{~K}$ ). In the case of CC-like simulations, velocities $>2 \mathrm{kms}^{-1}$ generate bulk $T>700 \mathrm{~K}$ (evidence for metamorphism at these temperatures is extremely rare in CCs). In the case of OC-like simulations, velocities $>1.25 \mathrm{~km} \mathrm{~s}^{-1}$ (corresponding to bulk $T>600 \mathrm{~K}$ ) generate matrix $T$ (final) such that $>20 \%$ of matrix is heated above the solidus (OC meteorites do not show evidence for widespread matrix melting). To translate our mesoscale observations into a constraint on asteroid-scale impact velocity, we can go to our macroscale numerical simulations of planetesimal collisions (Figs 3 and 4) which account for shock-wave attenuation with distance. Material in the range $400 \mathrm{~K}<$ bulk $T$ (final) $<700 \mathrm{~K}$ (blue) is consistent with meteorite data, according to the mesoscale simulation predictions. Material that experienced bulk $T$ (final) $>700 \mathrm{~K}$ (green and red) is not consistent with data from primitive unequilibrated meteorites. Of the material that is compacted to meteorite-like porosities, we assume that if $>20 \%$ experienced high bulk $T$ (final), and/or widespread matrix melting (for example, in OC targets), it would be observed in the meteorite record. In the example suite of simulations shown in Fig. 4 it is apparent that if asteroid collisional velocities exceeded $3 \mathrm{~km} \mathrm{~s}^{-1}$ in head-on impacts during the compaction phase (translating to $\sim 4 \mathrm{~km} \mathrm{~s}^{-1}$ for average $45^{\circ}$ impacts) this boundary would be crossed. Put another way, mesoscale simulations showing that velocities $>2 \mathrm{~km} \mathrm{~s}^{-1}$ for CC impacts and $>1.25 \mathrm{~km} \mathrm{~s}^{-1}$ for OC impacts are incompatible with meteorite data would translate to velocities of $3-4 \mathrm{~km} \mathrm{~s}^{-1}$ in real asteroid collisions.

Although the specific collisional 'speed limit' will depend on a variety of model assumptions, it is apparent (a) that initial impact velocities for OC and CC parent bodies were similar, and (b) that they must have been low. The chondrule age upper limit on accretion time, and chronometry for secondary olivine, indicates that these general constraints applied from $\sim 4 \mathrm{Myr}$ after solar system formation. In addition to relevance for understanding the early evolution of primitive solar system objects, our 'speed limit' has implications for dynamical models of giant planet migration. According to recent models ${ }^{45,46}$, OC-like parent bodies formed at 0.7-3 AU and CC-like bodies formed between the giant planets and from 8.0 to 13.0 AU. The migration of Jupiter resulted in an early (4-5 Myr) depletion and excitation of the asteroid belt region $^{45,46}$. It was then repopulated with material from these two source regions ${ }^{45,46}$. Estimates for asteroid collisional velocities at $4 \mathrm{Myr}$ from models that do not involve early migration of Jupiter ${ }^{47}$ are $5-9 \mathrm{~km} \mathrm{~s}^{-1}$. Grand Tack ${ }^{45,46}$ variants would produce higher mean impact velocities. This is not consistent with our observations. In addition, if OCs and CCs are derived from very different source regions we would expect to see evidence of rather different initial impact velocities. We do not. With respect to the planetary dynamics of the early solar system, our speed limit constraint is consistent with a relatively 'late' phase of main-belt collisional evolution, rather than the early, rapid depletion of a massive main belt ${ }^{45,46}$.

\section{Methods}

Numerical modelling. The iSALE shock physics code ${ }^{17-19}$ was used in two ways to quantify the compaction of porous planetesimal material in an impact. In one suite of models, 2D plane-strain, 'mesoscale' simulations were performed of shock wave propagation through a bimodal mixture of non-porous disks (chondrules) surrounded by a highly porous continuous matrix. The purpose of these simulations was to quantify the heterogeneity in peak pressure, post-shock temperature and post-shock porosity caused by the propagation of a single shock wave through a heterogeneous bimodal 'chondritic' mixture. In another suite of models, 2D axially symmetric, 'macroscale' simulations were performed of head-on collisions between porous planetesimals of different sizes and at speeds of a few $\mathrm{km} \mathrm{s}^{-1}$. The purpose of these simulations was to constrain the post-impact distribution of compacted material consistent with meteoritic evidence.

Mesoscale simulations. Chondritic meteorites are comprised of (nominally) zero-porosity spherical chondrules $(0.1-1 \mathrm{~mm}$ in size) set in a highly porous matrix aggregate composed of sub- $\mu \mathrm{m}$ monomers. The three orders of magnitude difference in lengthscale of these two components allowed us to simulate shock in this material by explicitly resolving the chondrules as disks of non-porous forsterite in the $2 \mathrm{D}$ computational grid and surrounding these with a high-porosity continuum of forsterite (in 16 simulations), and serpentine (in 5). The porosity in this continuous matrix is parameterized as it exists on a lengthscale too small to be resolved in the simulation. The epsilon-alpha porous compaction model ${ }^{19,48}$ was used to parameterize the effect of pore space compaction during the shock, while an ANEOS equation of state table for dunite/forsterite ${ }^{20}$ was used to describe the thermodynamic response of the chondrule material. The non-porous part of the porous matrix material was described by either the dunite/forsterite equation of state, or an ANEOS equation of state table for serpentine ${ }^{21}$. A strength model for geologic materials ${ }^{18}$ was used to represent the response of the chondrules and matrix to changes in deviatoric stress: the chondrules were given a high cohesive strength $(1 \mathrm{GPa})$, whereas the porous matrix was assumed to be very weak, with a cohesive strength of $100 \mathrm{kPa}$. A complete listing of material parameters used for the chondrules and matrix is given in Table 2. To generate a bimodal mixture analogous to the chondrule/matrix mixtures in chondritic meteorites, we randomly placed chondrule analogues (dunite disks) of various sizes ranging from 0.3 to $1 \mathrm{~mm}$ in diameter into an otherwise continuous region of the porous matrix analogue until the desired chondrule volume fraction was achieved. All simulations assumed an initial temperature of $300 \mathrm{~K}$ and an initial matrix porosity of $70 \%$, apart from selected comparison runs at 60 and $80 \%$. The remaining initial conditions are summarized in Table 2.

In the mesoscale simulations, numerical planar impact experiments were performed in which a flyer plate impacted a target, comprising a sample sandwiched between a cover plate above and a buffer plate below (Fig. 6). The flyer, cover, sample and buffer plates were all comprised of the same bimodal mixture of non-porous chondrule disks, surrounded by a high-porosity matrix. The presence of a cover plate allowed the planar shock wave to achieve a steady form before passing through the sample and then the adjacent buffer plate. The simulation time extended until the sample was released from high pressure by a release wave from the rear of the flyer plate (see Fig. 6).

Figure 6 illustrates the propagation of the shock wave from a representative numerical simulation. Upon impact an upward moving shock wave is generated in the flyer plate and a downward shock wave is generated in the cover plate. As the shock propagates though the cover plate it evolves to a steady wave as the shock front thickness and rise time increase to constant values determined by the mesoscale structure of the material. For the particle size, bulk porosity and shock pressure range in our simulations; the shock front thickness was $\sim 1-3$ chondrule diameters, consistent with front thicknesses determined by mesoscale simulations of granular material compaction ${ }^{49}$. A consequence of the initial increase in shock front thickness is that shock compaction is greatest at the impact plane and decays with distance until a steady wave is achieved. This is evident from the gradient in porosity in the cover and flyer plates near the interface between them. The thickness of the cover plate (a few chondrule diameters) was chosen to ensure that the shock wave was steady when it entered the sample; no gradient in compaction exists within the sample. The mesoscale structure also creates resonant oscillations around the steady wave amplitude, which result in the important heterogeneous temperatures within the sample that are the focus of our study. Such oscillations have been observed in laboratory experiments of layered composites of 'hard' and 'soft' materials ${ }^{50}$ and observed and modelled in porous granular materials ${ }^{51}$ and porous rocks ${ }^{52}$. The magnitude and duration of the oscillations depends on the 
impedance mismatch between the components in the system ${ }^{50}$, which is very large for the chondrule/matrix system studied here. It is important to note that while the duration of the shock in our numerical simulations is long compared with typical laboratory experiments, it is short compared with shock durations in $\mathrm{km}$-scale impacts on planetesimals (the shock duration is appropriate for $\mathrm{cm}$-scale impactors). However, a systematic verification study that varied the shock duration showed no significant effect of shock duration on either the mean or the variance of the shock temperatures recorded in the simulations, provided the shock duration was longer than the time necessary to achieve a steady wave. Hence, extrapolation of results to longer shock durations is justified. To record changes in temperature, pressure and distension (porosity), passive tracer particles were initially placed one per cell and subsequently followed the particle path of that material. Both peak and instantaneous pressure and temperature were recorded by the tracers, as well as the instantaneous matrix distension $(1 /(1-$ porosity $))$. Bulk temperature, pressure and distension were computed as volume-weighted averages of all tracers in the shocked sample plate. Note that a consequence of the heterogeneity in shock propagation is that the peak shock pressure recorded by each tracer throughout the duration of shock wave passage can be substantially higher than the instantaneous bulk shock pressure at any time. For example, in the simulation shown in Fig. $1 \mathrm{~b}$ the instantaneous bulk shock pressure in the shock wave was approximately $3 \pm 0.3 \mathrm{GPa}$ and yet the mean peak shock pressure experienced by chondrule and matrix material in the sample was $6.5 \pm 1.8$ and $8.4 \pm 2.6 \mathrm{GPa}$, respectively. Variations in peak and post-shock state were visualized using contour plots (for example, Fig. 1) and cross-sections through the sample at specific time intervals

In post-processing, the statistical data from the tracer particles were analysed to obtain the mean and s.d. of each variable in both the chondrule and the matrix analogues (see Table 1). Values of post-shock temperature and porosity were recorded just after the release wave had passed through the sample mixture (for example, $\sim 50 \mu$ s in Fig. 6). Repeat simulations with the same input parameters but different resolutions (cells across the largest chondrule) and different random distributions of chondrules were performed. In all cases, changes in the mean values were well within the s.d. of that value in a single simulation. To reflect the variability in mean values for simulations with different particle distributions, values in Table 1 are given to three significant figures only.

Figure 7 illustrates the variation in the bulk shock response, as well as in the response of the matrix and chondrule components, with impact velocity. The peak shock pressure experienced by both the matrix and chondrules can be more than twice the bulk shock pressure of the steady shock wave. The massive difference in compressibility between the porous matrix and the non-porous chondrules results in large temperature differences between the matrix and chondrules. Impact velocities $<4 \mathrm{~km} \mathrm{~s}^{-1}$ are not sufficient to compact all porosity from the matrix/ chondrule mixture.

We note that the agreement between the macroscale parameterization of porous compaction (the computed Hugoniot; black solid lines in Fig. 7) and the bulk behaviour observed in the mesoscale simulations (black circles) demonstrates self-consistency between the mesoscale modelling approach and the bulk parameterization used in previous work ${ }^{53}$ and the macroscale models of impacts on a planetesimals (see macroscale modelling section). The difference in average peak shock pressure between the chondrules and matrix is a consequence of the large strength difference between the two components. While the average peak longitudinal stress is the same in the chondrule and matrix components, the much higher strength of the chondrules implies that the corresponding peak pressure (the isotropic part of the stress tensor) in the chondrule is lower than in the weaker matrix.

The mesoscale simulations described here provide a significant first step in quantifying the heterogeneous response of chondritic precursor material to shock compaction, which we will refine in future by addressing some of the simplifying assumptions used here. Principal among these is the use of $2 \mathrm{D}$ plane-strain geometry, rather than a more realistic three-dimensional (3D) geometry. This assumption was necessary to limit computational cost. In 3D geometry, out-ofplane contacts between chondrules would likely stiffen the bulk response of the mixture, particularly in scenarios where the initial chondrule volume fraction is high. However, based on similar numerical mesoscale studies of pore-space compaction in $2 \mathrm{D}$ and $3 \mathrm{D}^{52}$ we expect qualitatively similar behaviour in both geometries, particularly with regard to the magnitude and lengthscale of the PT heterogeneity and the trends in PT heterogeneity with both impact velocity and initial matrix fraction.

The porous compaction model and the dunite/forsterite and serpentine equation of state tables used to describe both the chondrules and solid-component of the porous matrix are also oversimplified. The compaction model assumes that all of the pressure-volume work deposited by the shock in the porous matrix leads to increase in temperature. In reality, dissipative processes during compaction, such as grain deformation and fracturing lead to an increase in entropy as well as temperature. Neglecting the entropy increase during crushing will result in an overestimate of shock heating, but this is difficult to quantify without experimental measurements of shock heating. The version of ANEOS used to derive the table does not permit both solid-solid and solid-liquid phase transitions to be included at the same time ${ }^{54}$. As in previous work ${ }^{53}$ the effect of the latter was regarded as less important than that of the former. Neglecting latent heat of melting implies that temperatures in the table that exceed the solidus are overestimated. In addition, as the real matrix is a multicomponent system, the temperature increase in the matrix may be buffered by one of the less refractory components reaching the point of a phase change (for example, melting or vaporization) below the assumed melting point of our single-component chondritic analogue $(1,373 \mathrm{~K})$. As a first step to address this issue, we performed a complementary suite of mesoscale simulations that used a serpentine equation of state to represent the solid component of the matrix in place of forsterite (see Table 1). In low-velocity simulations there is little difference in matrix $T$ (final) between serpentine and forsterite. In simulations $\geq 2 \mathrm{~km} \mathrm{~s}^{-1}$ temperature is buffered by phase changes in serpentine, reducing matrix $T$ (final). However, still evident are high matrix temperatures alongside 'cold' chondrules, and significant PT heterogeneity over short lengthscales.

At higher shock pressures, shock compression experiments of quart ${ }^{55}$ suggest that ANEOS overestimates the temperature increase and underestimates the entropy increase during shock compression, because it assumes a heat capacity in the fluid region that is too $\mathrm{low}^{56}$. If this limitation of ANEOS is also important for other silicate rocks, it implies that the shock pressure required to vaporize the matrix is overestimated by ANEOS and that peak- and post-shock temperatures above the liquids are also overestimated. As our primary focus here is relatively low-velocity collisions, which causes matrix heating below and up to the solidus, this limitation of ANEOS is of minor significance to our conclusions.

For the reasons described above, the peak- and post-shock temperatures quoted in Table 1, particularly those above the solidus, can be considered as upper limits for the given impact scenario. On the other hand, peak- and post-shock temperature are a strong function of initial temperature. A less conservative initial temperature in our models could easily compensate for any overestimate in temperature due to inadequacies of the material model. Moreover, the relative trends of increasing temperature with impact velocity and chondrule volume fraction are robust.

Of the assumed model parameters, peak- and post-shock matrix temperatures are most sensitive to the initial porosity of the matrix. Hence, the effect of initial matrix porosity on post-shock temperature provides a good measure of the sensitivity of the temperature estimates to all model assumptions. In simulations that varied the initial matrix porosity between 60 and $80 \%$, we observed increasing post-shock matrix temperature with increasing initial porosity, for the same impact velocity (Table 1, main text). However, as demonstrated in Table 1, the mean postshock matrix temperature for $60 \%$ initial matrix porosity is still $80-70 \%$ of the $80 \%$ initial matrix porosity values (at $1 \mathrm{~km} \mathrm{~s}^{-1}$ and $2 \mathrm{~km} \mathrm{~s}^{-1}$, respectively). Hence, temperature excursions are unlikely to be overestimated by $>20-30 \%$ in our current simulations. Comparing the results of mesoscale simulations for the same $70 \%$ initial matrix porosity, but different impact velocities, a matrix temperature increase of $20-30 \%$ corresponds to an impact velocity increase of $<1 \mathrm{~km} \mathrm{~s}^{-1}$. With regard to the 'speed limit' constraint provided by the lack of evidence for high matrix temperatures among meteorites, the uncertainty in matrix temperatures associated with a given mesoscale compaction scenario translates into an uncertainty in impact velocity of $<1 \mathrm{~km} \mathrm{~s}^{-1}$.

Macroscale simulations. In addition, a suite of 'macroscale' planetesimal collision simulations were performed. In macroscale simulations of planetesimal collisions, practical limits on computational mesh resolution did not allow us to explicitly resolve chondrule-scale heterogeneity in the planetesimals. Instead the material model of the chondrule-matrix mixture was approximated using the same approach as for the matrix material in the mesoscale simulations, but with strength and porosity parameters appropriate for the bulk material (see the 'Bulk' column of Table 2). Target planetesimals 200 and $500 \mathrm{~km}$ in diameter were modelled, with a uniform initial temperature $(300 \mathrm{~K})$. Two planetesimal bulk porosities were considered: $20 \%$, which is appropriate for an ordinary chondrite (OC) parent body; and $50 \%$, appropriate for a CC parent body. Impacting planetesimals of 10-150 km in diameter and with identical material properties to the target collided with the target at $1-10 \mathrm{~km} \mathrm{~s}^{-1}$.

In an impact on a real planetesimal, the shock wave attenuates rapidly with distance, but the peak-shock PT conditions are similar to the planar impact scenario. Tracer particles tracked planetesimal material exposed to different bulk post-shock temperatures (for example, Fig. 3) for comparison with the mesoscale simulation results and meteoritic constraints. The simplified material model used in our mesoscale simulations may overestimate the temperature excursions by as much as $20-30 \%$ (see supporting material), implying that an increase in impact velocity of up to $1 \mathrm{~km} \mathrm{~s}^{-1}$ (relative to those documented in Table 1) might be required to generate specific PT conditions.

Electron microscopy. We studied the composition and mineralogy of matrix, opaque assemblages and chondrules. A JEOL JXA-8530 field emission gun 'Hyperprobe' was used to collect quantitative X-ray elemental maps and point analyses for $\mathrm{Na}, \mathrm{Mg}, \mathrm{Al}, \mathrm{Si}, \mathrm{P}, \mathrm{S}, \mathrm{K}, \mathrm{Ca}, \mathrm{Fe}, \mathrm{Ni}$ and $\mathrm{Co}$ from a thin section using a $0.26-\mu \mathrm{m}$ beam. Regions of matrix with distinct average chemical composition were identified. Broad-beam analyses of these regions were also obtained. These regions were also examined by FE-SEM to establish porosity, grain distribution, size and morphology.

TEM sections were prepared using a FEI Quanta 200 3D dual beam FIB workstation. FIB-TEM sections were examined using a JEOL 2010F field-emission gun TEM/scanning TEM equipped with an ultrathin window X-ray EDS. Four FIB sections were extracted from areas where the bulk composition and/or the porosity 
drastically changed. In addition, four other FIB sections from opaque assemblages were available from a previous study. Acquisition and processing of digital TEM images were conducted using GATAN's Digital Micrograph imaging software. Quantification of EDS data was performed using the Cliff-Lorimer thin-film approximation. EDS spectra were collected in the scanning TEM configuration, using a $0.6-\mathrm{nm}$ probe diameter. A counting time of $100 \mathrm{~s}$ was used to minimize the effects of beam damage and drift, while retaining meaningful counting statistics. After collecting each data point, each grain was examined using bright-field imaging. If the grain was not stable under the EDS probe for the 100-s duration of the analysis (that is, analysis resulted in a hole in the sample), the resulting analysis was not included in the data set. These analyses were excluded because it was unclear whether there was a beam overlap between the phase of interest and phase(s) lying underneath. Compositional heterogeneities were correlated with mineralogical markers, such as disequilibrium mineralogical assemblages, temperature-related crystallographic transitions, thermal decomposition of mineral phases and extent of solid solutions, to determine the scale of temperature heterogeneity.

FE-EPMA was carried out at the Regional Microanalytical and Imaging Center at Fayetteville State University. FIB and TEM analyses were conducted at the Material Characterization Laboratory at Pennsylvania State University.

\section{References}

1. Stöffler, D., Keil, K. \& Scott, E. R. D. Shock metamorphism of ordinary chondrites. Geochim. Cosmochim. Acta 55, 3845-3867 (1991).

2. Scott, E. R. D., Keil, K. \& Stöffler, D. Shock metamorphism of carbonaceous chondrites. Geochim. Cosmochim. Acta 56, 4281-4293 (1992).

3. Sharp, T. G. \& DeCarli, P. S. in Meteorites and the Early Solar System II (eds Lauretta, D. S. \& McSween, Jr. H. Y.) (Univ. Arizona Press, 653-677, 2006).

4. Kieffer, S. W., Phakey, P. P. \& Christie, J. M. Shock processes in porous quartzite: transmission electron microscope observations and theory. Contrib. Mineral. Petrol. 59, 41-93 (1976).

5. Blum, J. Grain growth and coagulation. Astrophys. Dust ASP Conf. Ser. 309, 369-391 (2004)

6. Ormel, C. W., Cuzzi, J. N. \& Tielens, A. G. G. M. Co-accretion of chondrules and dust in the solar nebula. Astrophys. J. 679, 1588-1610 (2008).

7. Bland, P. A. et al. Earliest rock fabric formed in the Solar System preserved in a chondrule rim. Nat. Geosci. 4, 244-247 (2011).

8. Beitz, E., Güttler, C., Nakamura, A. M., Tsuchiyama, A. \& Blum, J. Experiments on the consolidation of chondrites and the formation of dense rims around chondrules. Icarus 225, 558-569 (2013).

9. Topel-Schadt, J. \& Müller, W. F. The submicroscopic structure of the unequilibrated ordinary chondrites Chainpur, Mezo-Madaras amd Tieschitz: a transmission electron-microscope study. Earth Planet. Sci. Lett. 74, 1-12 (1985).

10. Abreu, N. M., Bland, P. A. \& Rietmeijer, F. J. M. Effects of shock metamorphism on the matrix of CR chondrites: GRA 06100. Lunar Planet. Sci. Conf. 45 abstract \#2753 (2014).

11. Davison, T. M., Ciesla, F. J. \& Collins, G. S. Post-impact thermal evolution of porous planetesimals. Geochim. Cosmochim. Acta 95, 252-269 (2012).

12. Consolmagno, G. J., Britt, D. T. \& Stoll, C. P. The porosities of ordinary chondrites: models and interpretation. Meteorit. Planet. Sci. 33, 1221-1229 (1998).

13. Sasso, M. R. et al. Incompletely compacted equilibrated ordinary chondrites. Meteorit. Planet. Sci. 44, 1743-1753 (2009).

14. Macke, R. J., Consolmagno, G. J. \& Britt, D. T. Density, porosity, and magnetic susceptibility of carbonaceous chondrites. Meteorit. Planet. Sci. 46, 1842-1862 (2011).

15. Watt, L. E., Bland, P. A., Prior, D. J. \& Russell, S. S. Fabric analysis of Allende matrix using EBSD. Meteorit. Planet. Sci. 41, 989-1001 (2006).

16. Weidenschilling, S. J. \& Cuzzi, J. N. in Meteorites and the Early Solar System II (eds Lauretta, D. S. \& McSween, Jr. H. Y.) (Univ. Arizona Press, 473-485, 2006)

17. Amsden, A. A., Ruppel, H. M. \& Hirt, C. W. SALE: Simplified ALE Computer Program for Fluid Flow at all Speeds, LA-8095 (Los Alamos National Laboratory, pp 101, 1980)

18. Collins, G. S., Melosh, H. J. \& Ivanov, B. A. Modeling damage and deformation in impact simulations. Meteorit. Planet. Sci. 39, 217-231 (2004).

19. Wünnemann, K., Collins, G. S. \& Melosh, H. J. A strain-based porosity model for use in hydrocode simulations of impacts and implications for transient crater growth in porous targets. Icarus 180, 514-527 (2006).

20. Benz, W., Cameron, A. G. W. \& Melosh, H. J. The origin of the Moon and the single-impact hypothesis III. Icarus 81, 113-131 (1989).

21. Brookshaw, L. in Working Paper Series SC-MC-9813 (Faculty of Sciences, Univ. Southern Queensland, 1998).

22. Leroux, $\mathrm{H}$. Microstructural shock signatures of major minerals in meteorites Eur. J. Mineral 13, 53-272 (2001).

23. Tomeoka, K., Yamahana, Y. \& Sekine, T. Experimental shock metamorphism of the Murchison CM carbonaceous chondrite. Geochim. Cosmochim. Acta 63, 3683-3703 (1999)
24. Brigatti, M. F. \& Poppi, L. Natural and monoionic aliettite: hydration and dehydration states. Clay Min. 22, 187-197 (1987).

25. Anovitz, L. M. \& Essene, E. J. Phase equilibria in the system $\mathrm{CaCO}_{3}-\mathrm{MgCO}_{3}$ $\mathrm{FeCO}_{3}$. J. Petrol. 28, 389-415 (1986).

26. Schmid-Beurmann, P., Cemic, L. \& Knitter, St. Crystal chemical properties of synthetic lazulite-scorzalite solid-solution series. Phys. Chem. Miner. 26, 496-505 (1999)

27. Kimura, M., Grossman, J. N. \& Weisberg, M. K. Fe-Ni metal and sulfide minerals in CM chondrites: an indicator for thermal history. Meteorit. Planet. Sci. 46, 431-442 (2011).

28. Pierazzo, E. \& Melosh, H. J. Melt production in oblique impacts. Icarus 145, 252-261 (2000).

29. Cooper, R. F. \& Kohlstedt, D. L. Sintering of olivine and olivine-basalt aggregates. Phys. Chem. Miner. 11, 5-16 (1984).

30. Weiss, B. P., Gattacceca, J., Stanley, S., Rochette, P. \& Christensen, U. R. Paleomagnetic records of meteorites and early planetesimal differentiation. Space Sci. Rev. 152, 341-390 (2010).

31. Crawford, D. A. \& Schultz, P. H. Electromagnetic properties of impactgenerated plasma, vapour and debris. Int. J. Imp. Eng. 23, 169-180 (1999).

32. Kojima, T., Lauretta, D. S. \& Buseck, P. R. Accretion, dispersal, and reaccumulation of the Bishunpur (LL3.1) brecciated chondrite: evidence from troilite-silicate-metal inclusions and chondrule rims. Geochim. Cosmochim. Acta 67, 3065-3078 (2003).

33. Brenker, F. E. \& Krot, A. N. Late-stage, high-temperature processing in the Allende meteorite: record from $\mathrm{Ca}$,Fe-rich silicate rims around dark inclusions. Am. Miner. 89, 1280-1289 (2004).

34. Brenker, F. E., Palme, H. \& Klerner, S. Evidence for solar nebula signatures in the matrix of the Allende meteorite. Earth Planet. Sci. Lett. 178, 185-194 (2000).

35. Müller, W. F. Transmission electron microscopy study of some minerals of the meteorite Allende. 9th Lunar Planet. Sci. Conf. 609-610 (1978).

36. Caillet, C., MacPherson, G. J. \& Zinner, E. K. Petrologic and Al-Mg isotopic clues to the accretion of two refractory inclusions onto the Leoville parent body: One was hot, the other wasn't. Geochim. Cosmochim. Acta 57, 4725-4743 (1993).

37. Rubin, A. E. Postshock annealing and postannealing shock in equilibrated ordinary chondrites: Implications for the thermal and shock histories of chondritic asteroids. Geochim. Cosmochim. Acta 68, 673-689 (2004).

38. Amelin, Y. et al. U-Pb chronology of the Solar System's oldest solids with variable ${ }^{238} \mathrm{U} /{ }^{235} \mathrm{U}$. Earth Planet. Sci. Lett. 300, 343-350 (2010).

39. Connelly, J. N. et al. The absolute chronology and thermal processing of solids in the solar protoplanetary disk. Science 338, 651-655 (2012).

40. Connelly, J. N., Amelin, Y., Krot, A. N. \& Bizarro, M. Chronology of the solar system's oldest solids. Astrophys. J. 675, L121-L124 (2008).

41. Amelin, Y., Krot, A. N., Hutcheon, I. D. \& Ulyanov, A. A. Lead isotopic ages of chondrules and calcium-aluminum-rich inclusions. Science 297, 1678-1683 (2002).

42. Villeneuve, J., Chaussidon, M. \& Libourel, G. Homogeneous distribution of ${ }^{26} \mathrm{Al}$ in the Solar System and the Mg isotopic composition of chondrules. Science 325, 985-988 (2009).

43. Doyle, P. M., Nagashima, K., Jogo, K. \& Krot, A. N. ${ }^{53} \mathrm{Mn}-{ }^{53} \mathrm{Cr}$ chronometry reveals secondary fayalite in Asuka 881317 (CV3) and MacAlpine Hills 88107 (CO/CM-like) formed 4-5Ma after CV CAIs. Lunar Planet. Sci. Conf. 44 abstract \#1793 (2013).

44. Doyle, P. M., Krot, A. N., Nagashima, K., Dobrica, E. \& Brearley, A. J. Manganese-chromium ages of aqueous alteration of unequilibrated ordinary chondrites. Lunar Planet. Sci. Conf. 45 abstract \#1726 (2014).

45. Walsh, K. J., Morbidelli, A., Raymond, S. N., O’Brien, D. P. \& Mandell, A. M. A low mass for Mars from Jupiter's early gas-driven migration. Nature 475, 206-209 (2011).

46. Walsh, K. J., Morbidelli, A., Raymond, S. N., O’Brien, D. P. \& Mandell, A. M. Populating the asteroid belt from two parent source regions due to the migration of giant planets - "The Grand Tack". Meteorit. Planet. Sci. 47, 1941-1947 (2012).

47. Davison, T. M., O’Brien, D. P., Ciesla, F. J. \& Collins, G. S. The early impact histories of meteorite parent bodies. Meteorit. Planet. Sci. 48, 1894-1918 (2013).

48. Collins, G. S., Melosh, H. J. \& Wünnemann, K. Improvements to the epsilonalpha compaction model for simulating impacts into high-porosity solar system objects. Int. J. Impact Eng. 38, 434-439 (2011).

49. Benson, D. J., Nesterenko, V. F., Jonsdottir, F. \& Meyers, M. A. Quasistatic and dynamic regimes of granular material deformation under impulse loading. J. Mech. Phys. Solids 45, 1955-1999 (1997).

50. Zhuang, S. M., Ravichandran, G. \& Grady, D. E. W. An experimental investigation of shock wave propagation in periodically layered composites. J. Mech. Phys. Solids 51, 245-265 (2003).

51. Trott, W. M., Baer, M. R., Castaneda, J. N., Chhabildas, L. C. \& Asay, J. R. Investigation of the mesoscopic scale response of low-density pressings of granular sugar under impact. J. Appl. Phys. 101, 024917-024921 (2007). 
52. Güldemeister, N., Wünnemann, K., Durr, N. \& Hiermaier, S. Propagation of impact-induced shock waves in porous sandstone using mesoscale modeling. Meteorit. Planet. Sci. 48, 115-133 (2013).

53. Davison, T. M., Collins, G. S. \& Ciesla, F. J. Numerical modeling of heating in porous planetesimal collisions. Icarus 208, 468-481 (2010).

54. Melosh, H. J. A hydrocode equation of state for $\mathrm{SiO}_{2}$. Meteorit. Planet. Sci. 42, 2079-2098 (2007)

55. Hicks, D. G. et al. Dissociation of liquid silica at high pressures and temperatures. Phys. Rev. Lett. 97, 025502 (2002).

56. Kraus, R. G. et al. Shock vaporization of silica and the thermodynamics of planetary impact events. J. Geophys. Res. 117, E004082 (2012).

57. Wünnemann, K., Collins, G. S. \& Osinski, G. R. Numerical modeling of impact melt production in porous rocks. Earth Planet. Sci. Lett. 269, 529-538 (2008).

\section{Acknowledgements}

This work was funded by the Australian Research Council via their Laureate Fellowship programme, the UK Science and Technology Facilities Council, the Royal Society and the UK Natural Environment Research Council. We thank Yuri Amelin and Jürgen Blum for useful discussions, and Anton Kearsley for Renazzo imagery. We also gratefully acknowledge the iSALE developers (www.isale-code.de), in particular Nicole Güldemeister.

\section{Author contributions}

P.A.B. and G.S.C. originated the study and collaborated to explore potential implications, iterating towards a model appropriate for simulating chondrite compaction, and generating outputs that offered predictions against the chondrite record. P.A.B. led interpretation of the meteorite record and comparison with numerical results and wrote much of the paper. G.S.C. designed the mesoscale numerical approach described in this work. T.M.D. performed much of the numerical modelling and model analysis. N.M.A. performed combined microscopy and microanalytical study, producing the isotherm map of GRA 06100. F.J.C. contributed intellectual input on both numerical modelling and the meteorite record. A.R.M. and J.M. contributed to models involving transient heating, and detail on implications for palaeomagnetism.

\section{Additional information}

Competing financial interests: The authors declare no competing financial interests.

Reprints and permission information is available online at http://npg.nature.com/ reprintsandpermissions/

How to cite this article: Bland, P. A. et al. Pressure-temperature evolution of primordial solar system solids during impact-induced compaction. Nat. Commun. 5:5451 doi: 10.1038/ncomms6451 (2014).

This work is licensed under a Creative Commons Attribution 4.0 International License. The images or other third party material in this article are included in the article's Creative Commons license, unless indicated otherwise in the credit line; if the material is not included under the Creative Commons license, users will need to obtain permission from the license holder to reproduce the material. To view a copy of this license, visit http://creativecommons.org/licenses/by/4.0/ 